\title{
THE FINITE PART OF SINGULAR INTEGRALS IN SEVERAL COMPLEX VARIABLES
}

\author{
WANG XIAOQIN
}

\begin{abstract}
A divergent integral can sometimes be handled by assigning to it as its value the finite part in the sense of Hadamard. This is done by expanding the integral over the complement of a symmetric neighborhood of a singularity in powers of the radius, and throwing away the negative powers. In this paper the finite part of a singular integral of Cauchy type is defined, and this is then used to describe the boundary behavior of derivatives of a Cauchy-type integral. The finite part of a singular integral of Bochner-Martinelli type is studied, and an extension of the Plemelj jump formulas is shown to hold.
\end{abstract}

\section{INTRODUCTION}

We know that an integral of the type

$$
\int_{a}^{b} \frac{f(x) d x}{(b-x)^{k+1 / 2}}
$$

is divergent if $k \geq 1 / 2$. For $k$ an integer $\geq 1$, Hadamard [1952] derived an expression which he called the finite part of (1.1), and which, as he showed, possesses many important properties. His definition is

$$
\begin{aligned}
& \text { FP } \int_{a}^{b} \frac{f(x) d x}{(b-x)^{k+1 / 2}} \\
& \quad=\lim _{\varepsilon \rightarrow 0}\left[\int_{a}^{b-\varepsilon} \frac{f(x) d x}{(b-x)^{k+1 / 2}}-\sum_{j=0}^{k-1} \frac{(-1)^{j} f^{(j)}(b)}{j !(k-j-1 / 2) \varepsilon^{k-j-1 / 2}}\right] .
\end{aligned}
$$

Fox [1957] considered a divergent integral

$$
\int_{a}^{b} \frac{f(x) d x}{(x-u)^{k+1}}
$$

where $a<u<b$ and $k$ is a nonnegative integer. His definition is

$$
\mathrm{FP} \int_{a}^{b} \frac{f(x) d x}{(x-u)^{k+1}}=\lim _{\varepsilon \rightarrow 0}\left[\int_{a}^{u-\varepsilon} \frac{f(x) d x}{(x-u)^{k+1}}+\int_{u+\varepsilon}^{b} \frac{f(x) d x}{(x-u)^{k+1}}-H_{k}(u, \varepsilon)\right] \text {. }
$$

Received by the editors February 28, 1991.

1980 Mathematics Subject Classification (1985 Revision). Primary 32A25, 32F05; Secondary 45E05, 32B15.

Key words and phrases. Singular integral, Hadamard's finite part, Bochner-Martinelli kernel.

The author is partially supported by the Gustaf Sigurd Magnuson Foundation of the Royal Swedish Academy of Sciences. 
Here $H_{k}(u, \varepsilon)=0$ if $k=0$, and

$$
H_{k}(u, \varepsilon)=\sum_{j=0}^{k-1} \frac{f^{(j)}(u)}{j !}\left(\frac{1-(-1)^{k-j}}{(k-j) \varepsilon^{k-j}}\right),
$$

if $k$ is a positive integer. For a given function $f$ the finite part is obtained here by subtracting negative powers, if any, of $\varepsilon$ in an expansion of the integral over the interval $[a, b]$ minus the symmetric interval $[u-\varepsilon, u+\varepsilon]$. If $u=a$ or $u=b$, the finite part can also be defined, but it is somewhat less stable then for integer $k$.

So we shall call the right-hand side of (1.2) or (1.4) the finite part of the integral (1.1), (1.3) respectively; in the special case $k=0$ in (1.4), when there are no negative powers of $\varepsilon$, it is also known as the principal value, or Cauchy principal value. We shall use the notation FP $\int$ and PV $\int$, respectively. See Hörmander [1983:70] for a general definition.

Similarly we can define the finite part of a singular integral

$$
\oint_{C} \frac{f(z) d z}{(z-u)^{k+1}}, \quad u \in C
$$

where $C$ is a closed curve. We can get formulas corresponding to the Plemelj jump formulas.

This paper is aimed at studying singular integrals in $\mathbf{C}^{n}$. In $\S 2$ we define the finite part of a singular integral

$$
\int_{S} \frac{f(\xi)}{\left(1-v \bar{\xi}^{\prime}\right)^{n-\beta+k}} d s(\xi)
$$

and obtain the corresponding Plemelj jump formulas which describe the boundary behavior of the higher derivatives of a Cauchy-type integral. In $\S 3$ we define the finite part of the singular integral of Bochner-Martinelli type

$$
\int_{\Omega} f(z) \frac{z_{k}-w_{k}}{|z-w|^{2}} K_{2 n-1}(z, w), \quad w \in \Omega, k=1, \ldots, n,
$$

and obtain similar theorems.

This paper is finished under the guidance of my advisors, Professor Christer Kiselman and Professor Zhong Tongde. Professor L. A. Aizenberg read this paper carefully and gave me suggestions. I am very grateful for his help.

\section{FINITE PART OF SINGULAR INTEGRALS ON SPHERES}

In several complex variables we do not have a kernel with all the good properties of the Cauchy kernel-both domain invariant and holomorphy. However, for a given domain there may exist good kernels, and in the case of the ball we can use

$$
\frac{1}{\omega}\left(1-z \bar{\xi}^{\prime}\right)^{-n} d s(\xi) .
$$

Here $\xi=\left(\xi_{1}, \ldots, \xi_{n}\right), z=\left(z_{1}, \ldots, z_{n}\right)$ are points in $\mathbf{C}^{n}$, and we use matrix multiplication, the prime indicating the transpose of a matrix so that $z \bar{\xi}^{\prime}=$ $\sum z_{j} \bar{\xi}_{j}$ and $|z|=\sqrt{z \bar{z}^{\prime}}$. We define the kernel for $\xi$ on the unit sphere and $z$ in the closed unit ball. We shall use the notations

$$
B=\left\{z \in \mathbf{C}^{n} ; z \bar{z}^{\prime}<1\right\}, \quad S=\left\{z \in \mathbf{C}^{n} ; z \bar{z}^{\prime}=1\right\}
$$


for the open unit ball and its boundary, the unit sphere, respectively. Moreover $d s(\xi)$ denotes the area element of the unit sphere whose area is

$$
\int_{S} d s(\xi)=\omega=\omega_{2 n-1}=\frac{2 \pi^{n}}{\Gamma(n)} .
$$

Suppose $f$ is an integrable function on $S$. Then

$$
\frac{1}{\omega} \int_{S} \frac{f(\xi) d s(\xi)}{\left(1-z \bar{\xi}^{\prime}\right)^{n}}
$$

exists for all $z \in B$, because in this case the integrand has no singularity at all. For $z \in S$ the integral exists for a particular point $z$ if $|f(\xi)| \leq C|\xi-z|^{\alpha}$ for some $\alpha>0$. Indeed, without losing generality we may take $z=(1,0, \ldots, 0)$ and assume that supp $f \subset\left\{\xi \in S ; \xi_{1} \neq 0\right\}$. Then

$$
\begin{aligned}
\frac{|f(\xi)|}{\left|1-z \bar{\xi}^{\prime}\right|^{n}} & =\frac{|f(\xi)|}{\left.\left|\bar{\xi}_{1}\left(\xi_{1}-1\right)+\right| \xi_{2}\right|^{2}+\cdots+\left.\left|\xi_{n}\right|^{2}\right|^{n}} \\
& \sim \frac{|f(\xi)|}{|| 1-\left.\xi_{1}|+| \xi_{2}\right|^{2}+\cdots+\left.\left|\xi_{n}\right|^{2}\right|^{n}},
\end{aligned}
$$

so if we introduce local coordinates $(y, w)=\left(\left|1-\xi_{1}\right|, \xi_{2}, \ldots, \xi_{n}\right) \in \mathbf{R} \times \mathbf{C}^{n-1}$ on the unit sphere near $(1,0, \ldots, 0)$, the singularity of $f(\xi) /\left(1-z \bar{\xi}^{\prime}\right)^{n}$ is not worse than that of the locally integrable function $\left(y+|w|^{2}\right)^{-n+\alpha / 2}$.

Generally for every $z \in S$ and a Hölder-continuous function $f$, we can define the Cauchy principal value of $(2.1)$ as

$$
\mathrm{PV} \frac{1}{\omega} \int_{S} \frac{f(\xi)}{\left(1-v \bar{\xi}^{\prime}\right)^{n}} d s(\xi)=\lim _{\varepsilon \rightarrow 0} \frac{1}{\omega} \int_{S \cap\left\{\left|1-v \bar{\xi}^{\prime}\right|>\varepsilon\right\}} \frac{f(\xi)}{\left(1-v \bar{\xi}^{\prime}\right)^{n}} d s(\xi),
$$

and we have a Plemelj jump formula, as shown in Lemmas 2.1 and 2.2; see Gong [1982].

Lemma 2.1. Suppose $f$ is a Hölder-continuous function on $S$, i.e., that there exist numbers $\alpha$ with $0<\alpha \leq 1$ and $C$ such that for any $\xi, \eta \in S$ we have $|f(\xi)-f(\eta)| \leq C|\xi-\eta|^{\alpha}$. Then the Cauchy principal value exists and we have that

$$
\operatorname{PV} \frac{1}{\omega} \int_{S} \frac{f(\xi)}{\left(1-v \bar{\xi}^{\prime}\right)^{n}} d s(\xi)=\frac{1}{\omega} \int_{S} \frac{f(\xi)-f(v)}{\left(1-v \bar{\xi}^{\prime}\right)^{n}} d s(\xi)+\frac{1}{2} f(v), \quad v \in S
$$

Lemma 2.2 (Plemelj jump formula). Suppose $f$ is a Hölder-continuous function on the unit sphere $S$. If $z$ tends to $v$ from the interior and satisfies

$$
\frac{|z-v|}{\min _{u \in S}|u-z|}=\frac{d(z, v)}{d(z, S)} \leq M
$$

for some constant $M$, then we have

$$
\lim _{z \rightarrow v} \frac{1}{\omega} \int_{S} \frac{f(\xi)}{\left(1-z \bar{\xi}^{\prime}\right)^{n}} d s(\xi)=\mathrm{PV} \frac{1}{\omega} \int_{S} \frac{f(\xi)}{\left(1-v \bar{\xi}^{\prime}\right)^{n}} d s(\xi)+\frac{1}{2} f(v), \quad v \in S .
$$

Lemma 2.3. Suppose $f$ is a continuous function on some neighborhood of the unit sphere $S$ and $k$ times continuously differentiable, with support contained 
in the ball $B(p, 1 / 2)$ of center $p$ and radius $1 / 2$, i.e., $B(p, 1 / 2)=\{z \in$ $\left.\mathbf{C}^{n} ;|z-p|<1 / 2\right\}$, where $p=(1,0, \ldots, 0)$. Let $L$ be the operator defined by

$$
\begin{gathered}
(L f)(\xi)=\frac{1}{\bar{\xi}_{1}}\left[\xi_{1} \frac{\partial f}{\partial \xi_{1}}(\xi)-\bar{\xi}_{1} \frac{\partial f}{\partial \bar{\xi}_{1}}(\xi)+f(\xi)\right], \\
L^{j} f=L\left(L^{j-1} f\right), \quad L^{0}(f)=f .
\end{gathered}
$$

Then $(1)(L f)(\xi)$ is a continuous function on a neighborhood of $S$ and is $(k-1)$ times continuously differentiable, with $\operatorname{supp} L f \subset B(p, 1 / 2)$.

(2) Suppose that all kth partial derivatives of $f$ are Hölder continuous. Then all $(k-1)$ th partial derivatives of $L f$ are Hölder continuous.

(3) (Partial integration). Let $k$ be a positive integer and let $0 \leq \beta<1$. For any $z \in \bar{B}, z_{1} \neq 0$, and any surface $\Sigma \subset S, z \notin \bar{\Sigma}$, we have

$$
\begin{aligned}
& \int_{\Sigma} \frac{f(\xi)}{\left(1-z \bar{\xi}^{\prime}\right)^{n-\beta+k}} d s(\xi) \\
&=\frac{1}{(n-\beta+k-1)(n-\beta+k-2) \cdots(n-\beta) z_{1}^{k}} \int_{\Sigma} \frac{\left(L^{k} f\right)(\xi)}{\left(1-z \bar{\xi}^{\prime}\right)^{n-\beta}} d s(\xi) \\
&-\sum_{j=0}^{k-1} \frac{A_{n, \beta, k, j}}{z_{1}^{j+1}} \int_{\partial \Sigma} \frac{\left(L^{j} f\right)(\xi) d \xi_{2} \cdots d \xi_{n} d \bar{\xi}_{2} \cdots d \bar{\xi}_{n}}{\left(1-z \bar{\xi}^{\prime}\right)^{n-\beta+k-j-1 \bar{\xi}_{1}}}
\end{aligned}
$$

where $A_{n, \beta, k, j}$ denotes the constant

$$
A_{n, \beta, k, j}=\frac{1}{(n-\beta+k-1) \cdots(n-\beta+k-1-j) 2^{n-1} i^{n}} .
$$

In particular, when $z \in B, z_{1} \neq 0$, we can take $\Sigma=S$ and obtain

$$
\begin{aligned}
& \int_{S} \frac{f(\xi)}{\left(1-z \bar{\xi}^{\prime}\right)^{n-\beta+k}} d s(\xi) \\
& \quad=\frac{1}{(n-\beta+k-1)(n-\beta+k-2) \cdots(n-\beta) z_{1}^{k}} \int_{S} \frac{\left(L^{k} f\right)(\xi)}{\left(1-z \bar{\xi}^{\prime}\right)^{n-\beta}} d s(\xi) .
\end{aligned}
$$

Proof. Since $B\left(p, \frac{1}{2}\right) \subset\left\{z \in \mathbf{C}^{n} ; z_{1} \neq 0\right\}, L f$ is well defined on some neighborhood of $S$ and (1) and (2) follow from the definition of the operator $L$. We shall prove (3).

On the unit sphere $S$ we have

$$
\bar{\xi}_{1} d \xi_{1}+\cdots+\bar{\xi}_{n} d \xi_{n}+\xi_{1} d \bar{\xi}_{1}+\cdots+\xi_{n} d \bar{\xi}_{n}=0 .
$$

On the subset of $S$ where $\xi_{1} \neq 0$ we have, if $j \geq 2$,

$$
d \bar{\xi}_{1} \equiv-\frac{\xi_{j}}{\xi_{1}} d \bar{\xi}_{j} \bmod \left(d \xi_{1}, \ldots, d \xi_{n}, d \bar{\xi}_{2}, \ldots,\left[d \bar{\xi}_{j}\right], \ldots, d \bar{\xi}_{n}\right) .
$$

Here and in the sequel the notation $\left[d \bar{\xi}_{j}\right]$ means that $d \bar{\xi}_{j}$ shall be omitted. 
The area element can be expressed as follows:

$$
\begin{aligned}
d s(\xi) & =\frac{1}{2^{n-1} i^{n}} \sum_{j=1}^{n}(-1)^{j-1} \bar{\xi}_{j} d \xi_{1} \cdots d \xi_{n} d \bar{\xi}_{1} \cdots\left[d \bar{\xi}_{j}\right] \cdots d \bar{\xi}_{n} \\
& =\frac{1}{2^{n-1} i^{n} \xi_{1}} \sum_{j=1}^{n} \xi_{j} \bar{\xi}_{j} d \xi_{1} \cdots d \xi_{n} d \bar{\xi}_{2} \cdots d \bar{\xi}_{n} \\
& =\frac{1}{2^{n-1} i^{n} \xi_{1}} d \xi_{1} \cdots d \xi_{n} d \bar{\xi}_{2} \cdots d \bar{\xi}_{n} .
\end{aligned}
$$

Because of supp $f \subset B(p, 1 / 2)$ and

$$
d \xi_{1} \equiv-\xi_{1} d \bar{\xi}_{1} / \bar{\xi}_{1} \bmod \left(d \xi_{2}, \ldots, d \xi_{n}, d \bar{\xi}_{2}, \ldots, d \bar{\xi}_{n}\right)
$$

we get for any positive number $m$ :

$$
\begin{aligned}
& \int_{\Sigma} \frac{f(\xi)}{\left(1-z \bar{\xi}^{\prime}\right)^{m+1}} d s(\xi)=\int_{\Sigma \cap B(p, 1 / 2)} \frac{f(\xi)}{\left(1-z \bar{\xi}^{\prime}\right)^{m+1}} d s(\xi) \\
& =\frac{1}{2^{n-1} i^{n}} \int_{\Sigma \cap B(p, 1 / 2)} \frac{f(\xi)}{\xi_{1}\left(1-z \bar{\xi}^{\prime}\right)^{m+1}} d \xi_{1} \cdots d \xi_{n} d \bar{\xi}_{2} \cdots d \bar{\xi}_{n} \\
& =\frac{1}{2^{n-1} i^{n} m} \int_{\Sigma \cap B(p, 1 / 2)} \frac{f(\xi)}{\xi_{1}}\left(-\frac{\xi_{1}}{z_{1} \bar{\xi}_{1}}\right) d\left[\frac{1}{\left(1-z \bar{\xi}^{\prime}\right)^{m}}\right] d \xi_{2} \cdots d \xi_{n} d \bar{\xi}_{2} \cdots d \bar{\xi}_{n} \\
& =\frac{1}{2^{n-1} i^{n} m z_{1}} \int_{\Sigma \cap B(p, 1 / 2)} \frac{1}{\left(1-z \bar{\xi}^{\prime}\right)^{m}} d\left(\frac{f(\xi)}{\bar{\xi}_{1}}\right) d \xi_{2} \cdots d \xi_{n} d \bar{\xi}_{2} \cdots d \bar{\xi}_{n} \\
& -\frac{1}{2^{n-1} i^{n} m z_{1}} \int_{\partial(\Sigma \cap B(p, 1 / 2))} \frac{1}{\left(1-z \bar{\xi}^{\prime}\right)^{m}}\left(\frac{f(\xi)}{\bar{\xi}_{1}}\right) d \xi_{2} \cdots d \xi_{n} d \bar{\xi}_{2} \cdots d \bar{\xi}_{n} \\
& =\frac{1}{m z_{1}} \int_{\Sigma \cap B(p, 1 / 2)} \frac{1}{\left(1-z \bar{\xi}^{\prime}\right)^{m} \bar{\xi}_{1}}\left\{\xi_{1} \frac{\partial f}{\partial \xi_{1}}(\xi)-\bar{\xi}_{1} \frac{\partial f}{\partial \bar{\xi}_{1}}(\xi)+f(\xi)\right\} \\
& \cdot \frac{1}{2^{n-1} i^{n} \xi_{1}} d \xi_{1} \cdots d \xi_{n} d \bar{\xi}_{2} \cdots d \bar{\xi}_{n} \\
& -\frac{1}{2^{n-1} i^{n} m z_{1}} \int_{\partial(\Sigma \cap B(p, 1 / 2))} \frac{1}{\left(1-z \bar{\xi}^{\prime}\right)^{m}}\left(\frac{f(\xi)}{\bar{\xi}_{1}}\right) d \xi_{2} \cdots d \xi_{n} d \bar{\xi}_{2} \cdots d \bar{\xi}_{n} \\
& =\frac{1}{m z_{1}} \int_{\Sigma \cap B(p, 1 / 2)} \frac{(L f)(\xi)}{\left(1-z \bar{\xi}^{\prime}\right)^{m}} d s(\xi) \\
& -\frac{1}{2^{n-1} i^{n} m z_{1}} \int_{\partial(\Sigma \cap B(p, 1 / 2))} \frac{1}{\left(1-z \bar{\xi}^{\prime}\right)^{m}}\left(\frac{f(\xi)}{\bar{\xi}_{1}}\right) d \xi_{2} \cdots d \xi_{n} d \bar{\xi}_{2} \cdots d \bar{\xi}_{n} \\
& =\frac{1}{m z_{1}} \int_{\Sigma} \frac{(L f)(\xi)}{\left(1-z \bar{\xi}^{\prime}\right)^{m}} d s(\xi) \\
& -\frac{1}{2^{n-1} i^{n} m z_{1}} \int_{\partial \Sigma} \frac{1}{\left(1-z \bar{\xi}^{\prime}\right)^{m}}\left(\frac{f(\xi)}{\bar{\xi}_{1}}\right) d \xi_{2} \cdots d \xi_{n} d \bar{\xi}_{2} \cdots d \bar{\xi}_{n} .
\end{aligned}
$$

Now (3) follows by iteration.

In view of Lemma 2.3 it is reasonable to give the following definition. 
Definition 2.4. For any function $f$ which is $k-1$ times continuously differentiable on some neighborhood of $S, k \geq 1$, and any number $\beta$ with $0 \leq \beta<1$ we define the finite part of a singular integral

$$
\frac{1}{\omega} \int_{S} \frac{f(\xi)}{\left(1-\bar{\xi}_{1}\right)^{n-\beta+k}} d s(\xi)
$$

by

$$
\begin{aligned}
\mathrm{FP} & \frac{1}{\omega} \int_{S} \frac{f(\xi)}{\left(1-\bar{\xi}_{1}\right)^{n-\beta+k}} d s(\xi) \\
= & \lim _{\varepsilon \rightarrow 0}\left\{\frac{1}{\omega} \int_{S \cap\left\{\left|1-\bar{\xi}_{1}\right| \geq \varepsilon\right\}} \frac{f(\xi)}{\left(1-\bar{\xi}_{1}\right)^{n-\beta+k}} d s(\xi)\right. \\
& \left.\quad+\frac{1}{\omega} \sum_{j=0}^{k-1} A_{n, \beta, k, j} \int_{S \cap\left\{\left|1-\bar{\xi}_{1}\right|=\varepsilon\right\}} \frac{L^{j} f(\xi) d \xi_{2} \cdots d \xi_{n} d \bar{\xi}_{2} \cdots d \bar{\xi}_{n}}{\left(1-\bar{\xi}_{1}\right)^{n-\beta+k-j-1} \bar{\xi}_{1}}\right\},
\end{aligned}
$$

provided the limit exists. Here $A_{n, \beta, k, j}$ are the constants defined by (2.2).

Theorem 2.5. (1) Suppose $f$ is a continuous function on some neighborhood of $S$ and is continuously differentiable up to order $k$ with Hölder-continuous derivatives. Then the finite part in Definition 2.4 exists.

(2) There exist constants $c_{j}, j=0, \ldots, k-1$, such that

$$
\begin{aligned}
& \sum_{j=0}^{k-1} A_{n, \beta, k, j} \int_{S \cap\left\{\left|1-\bar{\xi}_{1}\right|=\varepsilon\right\}} \frac{\left(L^{j}(f)\right)(\xi) d \xi_{2} \cdots d \xi_{n} d \bar{\xi}_{2} \cdots d \bar{\xi}_{n}}{\left(1-\bar{\xi}_{1}\right)^{n-\beta+k-j-1} \bar{\xi}_{1}} \\
& =\sum_{j=0}^{k-1} \frac{c_{j}}{\varepsilon^{k-j-\beta}}+O\left(\varepsilon^{\beta}\right),
\end{aligned}
$$

provided that $f$ is $2 k$ times differentiable. Therefore the finite part of a singular integral is obtained by subtracting the negative powers of $\varepsilon$ from an expansion of the integral over the complement of a symmetric neighborhood of the singularity in the case $0<\beta<1$.

Proof. (1) Take an open covering $\left(U_{j}\right)_{1 \leq j \leq m}$ of $S$ such that $p \in U_{1}$ and $U_{1} \subset B(p, 1 / 2)$, and a partition of unit $\left(f_{j}\right)_{1 \leq j \leq m}$ subordinated to $\left(U_{j}\right)_{1 \leq j \leq m}$. Then we have $\sum_{j=1}^{m} f_{j}=1, \operatorname{supp}\left(f_{1} f\right) \subset U_{1}$, and $\operatorname{supp}\left(f_{j} f\right) \subset \mathbf{C}^{n} \backslash B(p, \sigma)$, where $2 \leq j \leq m$ and $\sigma$ is a positive number smaller than $1 / 2$.

Let $f^{\prime}=\sum_{j=2}^{m} f_{j} f$; then $\operatorname{supp} f^{\prime} \subset \mathbf{C}^{n} \backslash B(p, \sigma)$. If $\varepsilon$ is small enough, we have that

$$
\begin{aligned}
& \int_{S \cap\left\{\left|1-\bar{\xi}_{1}\right|>\varepsilon\right\}} \frac{f(\xi)}{\left(1-\bar{\xi}_{1}\right)^{n-\beta+k}} d s(\xi)=\int_{S \cap\left\{\left|1-\bar{\xi}_{1}\right| \geq \varepsilon\right\}} \frac{\sum_{j=1}^{m} f_{j} f}{\left(1-\bar{\xi}_{1}\right)^{n-\beta+k}} d s(\xi) \\
& \quad=\int_{S \cap\left\{\left|1-\bar{\xi}_{1}\right| \geq \varepsilon\right\}} \frac{f^{\prime}(\xi)}{\left(1-\bar{\xi}_{1}\right)^{n-\beta+k}} d s(\xi)+\int_{S \cap\left\{\left|1-\bar{\xi}_{1}\right| \geq \varepsilon\right\}} \frac{f_{1}(\xi) f(\xi)}{\left(1-\bar{\xi}_{1}\right)^{n-\beta+k}} d s(\xi) \\
& \quad=\int_{S} \frac{f^{\prime}(\xi)}{\left(1-\bar{\xi}_{1}\right)^{n-\beta+k}} d s(\xi)+\int_{S \cap\left\{\left|1-\bar{\xi}_{1}\right| \geq \varepsilon\right\}} \frac{f_{1}(\xi) f(\xi)}{\left(1-\bar{\xi}_{1}\right)^{n-\beta+k}} d s(\xi) \\
&=I_{1}+I_{2}
\end{aligned}
$$


where

$$
I_{1}=\int_{S} \frac{f^{\prime}(\xi)}{\left(1-\bar{\xi}_{1}\right)^{n-\beta+k}} d s(\xi)
$$

and

$$
I_{2}=\int_{S \cap\left\{\left|1-\bar{\xi}_{1}\right| \geq \varepsilon\right\}} \frac{f_{1}(\xi) f(\xi)}{\left(1-\bar{\xi}_{1}\right)^{n-\beta+k}} d s(\xi) .
$$

Here $I_{1}$ is independent of $\varepsilon$ so $\lim I_{1}$ exists.

Since $f_{1} f$ satisfies the condition in Lemma 2.3 we have

$$
\begin{aligned}
I_{2}= & -\sum_{j=0}^{k-1} A_{n, \beta, k, j} \int_{S \cap\left\{\left|1-\bar{\xi}_{1}\right|=\varepsilon\right\}} \frac{\left(L^{j}\left(f_{1} f\right)\right)(\xi) d \xi_{2} \cdots d \xi_{n} d \bar{\xi}_{2} \cdots d \bar{\xi}_{n}}{\left(1-\bar{\xi}_{1}\right)^{n-\beta+k-j-1 \bar{\xi}_{1}}} \\
& +\frac{1}{(n-\beta+k-1) \cdots(n-\beta+1)(n-\beta)} \int_{S \cap\left\{\left|1-\bar{\xi}_{1}\right| \geq \varepsilon\right\}} \frac{\left(L^{k}\left(f_{1} f\right)\right)(\xi)}{\left(1-\bar{\xi}_{1}\right)^{n-\beta}} d s(\xi) .
\end{aligned}
$$

If $\varepsilon$ is small enough, we have $S \cap\left\{\left|1-\bar{\xi}_{1}\right| \leq \varepsilon\right\} \subset B(p, \sigma)$. Therefore we see that

$$
\left.f_{1}\right|_{S \cap\left\{\left|1-\bar{\xi}_{1}\right| \leq \varepsilon\right\}}=1 \text {, }
$$

and

$$
\left.L^{j}\left(f_{1} f\right)\right|_{S \cap\left\{\left|1-\bar{\xi}_{1}\right| \leq \varepsilon\right\}}=\left.L^{j} f\right|_{S \cap\left\{\left|1-\bar{\xi}_{1}\right| \leq \varepsilon\right\}}
$$

Now

$$
\begin{aligned}
I_{2}= & \frac{1}{(n-\beta+k-1) \cdots(n-\beta+1)(n-\beta)} \int_{S \cap\left\{\left|1-\bar{\xi}_{1}\right| \geq \varepsilon\right\}} \frac{\left(L^{k}\left(f_{1} f\right)\right)(\xi)}{\left(1-\bar{\xi}_{1}\right)^{n-\beta}} d s(\xi) \\
& -\sum_{j=0}^{k-1} A_{n, \beta, k, j} \int_{S \cap\left\{\left|1-\bar{\xi}_{1}\right|=\varepsilon\right\}} \frac{\left(L^{j} f\right)(\xi) d \xi_{2} \cdots d \xi_{n} d \bar{\xi}_{2} \cdots d \bar{\xi}_{n}}{\left(1-\bar{\xi}_{1}\right)^{n-\beta+k-j-1} \bar{\xi}_{1}}
\end{aligned}
$$

so

$$
\begin{aligned}
\operatorname{FP} \frac{1}{\omega} \int_{S} \frac{f(\xi)}{\left(1-\bar{\xi}_{1}\right)^{n-\beta+k}} d s(\xi) \\
=\lim _{\varepsilon \rightarrow 0}\left\{\frac{1}{\omega} \int_{S \cap\left\{\left|1-\bar{\xi}_{1}\right| \geq \varepsilon\right\}} \frac{f(\xi)}{\left(1-\bar{\xi}_{1}\right)^{n-\beta}} d s(\xi)\right. \\
\left.\quad+\frac{1}{\omega} \sum_{j=0}^{k-1} A_{n, \beta, k, j} \int_{S \cap\left\{\left|1-\bar{\xi}_{1}\right|=\varepsilon\right\}} \frac{\left(L^{k} f\right)(\xi) d \xi_{2} \cdots d \xi_{n} d \bar{\xi}_{2} \cdots d \bar{\xi}_{n}}{\left(1-\bar{\xi}_{1}\right)^{n-\beta+k-j-1} \bar{\xi}_{1}}\right\} \\
=\frac{1}{\omega} I_{1}+\lim _{\varepsilon \rightarrow 0} \frac{1}{\omega} \frac{1}{(n-\beta+k-1) \cdots(n-\beta)} \int_{S \cap\left\{\left|1-\bar{\xi}_{1}\right| \geq \varepsilon\right\}} \frac{\left(L^{k}\left(f_{1} f\right)\right)(\xi)}{\left(1-\bar{\xi}_{1}\right)^{n-\beta}} d s(\xi) .
\end{aligned}
$$

Because all partial derivatives of $f$ are Hölder continuous, all partial derivatives of $f_{1} f$ are Hölder continuous. According to Lemma 2.3, $L^{k}\left(f_{1} f\right)$ is Hölder continuous and so the above limit exists, which is a Cauchy principal in the case $\beta=0$; a convergent integral in case $0<\beta<1$.

(2) Let $S_{\varepsilon}=S \cap\left\{\left|1-\bar{\xi}_{1}\right|=\varepsilon\right\}$. Let

$$
\varphi_{j}(\varepsilon)=\int_{S_{\varepsilon}} \frac{g(\xi)}{\left(1-\bar{\xi}_{1}\right)^{n-\beta+j-1} \bar{\xi}_{1}} d \xi_{2} \cdots d \xi_{n} d \bar{\xi}_{2} \cdots d \bar{\xi}_{n},
$$


where $g$ is any $2 j$ times differentiable function. We shall prove that there exists a function $\varphi$ which is real analytic at the point $\varepsilon=0$ such that

$$
\varphi_{j}(\varepsilon)=\frac{\varphi(\varepsilon)}{\varepsilon^{j-\beta}}+O\left(\varepsilon^{\beta}\right) .
$$

Expand $g(\xi) / \bar{\xi}_{1}$ around the point $p$ as follows

$$
\frac{g(\xi)}{\bar{\xi}_{1}}=\sum_{s+t+|K|<2 j} C_{s, t, K}\left(1-\bar{\xi}_{1}\right)^{s}\left(1-\xi_{1}\right)^{t} \xi^{K}+O\left(|\xi-p|^{2 j}\right),
$$

where $K=\left(0,0, k_{2}, k_{\overline{2}}, \ldots, k_{n}, k_{\bar{n}}\right)$ and $\xi^{K}=\xi_{2}^{k_{2}} \bar{\xi}_{2}^{k_{\overline{2}}} \ldots \xi_{n}^{k_{n}} \bar{\xi}_{n}^{k_{\bar{n}}}$. Since for all $\xi \in S_{\varepsilon}$ we have

$$
|\xi-p|^{2}=2 \operatorname{Re}\left(1-\bar{\xi}_{1}\right) \leq 2\left|1-\bar{\xi}_{1}\right|,
$$

we see that

$$
\begin{gathered}
\int_{S_{\varepsilon}} \frac{|\xi-p|^{2 j}}{\left|1-\bar{\xi}_{1}\right|^{n-\beta+j-1}} d \xi_{2} \cdots d \xi_{n} d \bar{\xi}_{2} \cdots d \bar{\xi}_{n} \\
\leq C \int_{S_{\varepsilon}} \frac{d \xi_{2} \cdots d \xi_{n} d \bar{\xi}_{2} \cdots d \bar{\xi}_{n}}{\left|1-\bar{\xi}_{1}\right|^{n-\beta-1}} \leq C \varepsilon^{\beta} .
\end{gathered}
$$

Let $1-\bar{\xi}_{1}=\varepsilon e^{i \theta}$ on $S_{\varepsilon}$. In terms of the new coordinates $\left(\theta, \xi_{2}, \ldots, \xi_{n}\right), S_{\varepsilon}$ has the following representation

$$
S_{\varepsilon}=\left\{\left(1-\varepsilon e^{-i \theta}, \xi_{2}, \ldots, \xi_{n}\right) \in \mathbf{C}^{n} ;|\tilde{\xi}|^{2}=\varepsilon\left(e^{i \theta}+e^{-i \theta}-\varepsilon\right)\right\},
$$

where we write $\tilde{\xi}$ for the vector $\left(\xi_{2}, \ldots, \xi_{n}\right) ;|\tilde{\xi}|^{2}=\left|\xi_{2}\right|^{2}+\cdots+\left|\xi_{n}\right|^{2}$. We now sum over all $s, t$ and $K$ such that $0 \leq s<j,-n-2 j \leq t \leq-n+j$, $|K|<2 j$; this gives

$$
\begin{aligned}
\varphi_{j}(\varepsilon) & =\sum C_{s t K} \frac{\varepsilon^{s}}{\varepsilon^{n-\beta+j-1}} \int_{\mid \tilde{\xi}^{2}=e\left(e^{i \theta}+e^{-i \theta}-\varepsilon\right)} e^{i(t-\beta) \theta} \xi^{K} d \xi_{2} \cdots d \xi_{n} d \bar{\xi}_{2} \cdots d \bar{\xi}_{n}+O\left(\varepsilon^{\beta}\right) \\
& =\sum C_{s t K} \frac{\varepsilon^{s}}{\varepsilon^{j-\beta}} \int_{|\tilde{\xi}|^{2}=e^{i \theta}+e^{-i \theta}-\varepsilon} e^{i(t-\beta) \theta}\left(\varepsilon^{1 / 2} \xi\right)^{K} d \xi_{2} \cdots d \xi_{n} d \bar{\xi}_{2} \cdots d \bar{\xi}_{n}+O\left(\varepsilon^{\beta}\right) \\
& =\sum C_{s t K} \frac{\varepsilon^{s}}{\varepsilon^{j-\beta}} \int_{|\xi|^{2} \leq e^{i \theta}+e^{-i \theta}-\varepsilon} e^{i(t-\beta) \theta}\left(\varepsilon^{1 / 2} \xi\right)^{K} d \theta d \xi_{2} \cdots d \xi_{n} d \bar{\xi}_{2} \cdots d \bar{\xi}_{n}+O\left(\varepsilon^{\beta}\right) \\
& =\sum C_{s t K} \frac{\varepsilon^{s}}{\varepsilon^{j-\beta}} \int_{-\arccos \varepsilon}^{\arccos \varepsilon} e^{i(t-\beta) \theta} d \theta \int_{\left|\tilde{\xi}^{2}\right|^{2} \leq e^{i \theta}+\varepsilon^{-i \theta}-\varepsilon}\left(\varepsilon^{1 / 2} \xi\right)^{K} d \xi_{2} \cdots d \xi_{n} d \bar{\xi}_{2} \cdots d \bar{\xi}_{n}+O\left(\varepsilon^{\beta}\right) .
\end{aligned}
$$

When $|K|$ is an odd number we have

$$
\int_{|\tilde{\xi}|^{2} \leq e^{i \theta}+e^{-i \theta}-\varepsilon}\left(\varepsilon^{1 / 2} \xi\right)^{K} d \xi_{2} \cdots d \xi_{n} d \bar{\xi}_{2} \cdots d \bar{\xi}_{n}=0
$$

because of the symmetry of the ball. When $|K|$ is an even integer, say $2 k$, we have

$$
\begin{gathered}
\int_{-\arccos \varepsilon}^{\arccos \varepsilon} e^{i(t-\beta) \theta} d \theta \int_{|\tilde{\xi}|^{2} \leq e^{i \theta}+e^{-i \theta}-\varepsilon} \varepsilon^{k} \xi^{2 k} d \xi_{2} \cdots d \xi_{n} d \bar{\xi}_{2} \cdots d \bar{\xi}_{n} \\
=C \varepsilon^{k} \int_{-\arccos \varepsilon}^{\arccos \varepsilon} e^{i(t-\beta) \theta}\left(e^{i \theta}+e^{-i \theta}-\varepsilon\right)^{n-1+k} d \theta
\end{gathered}
$$

which is a real-analytic function of $\varepsilon$. 
Theorem 2.6. Let the hypotheses be the same as those of Theorem 2.5. The Plemelj jump formulas hold, i.e., if $z$ tends to $p$ from the interior and satisfies $d(z, p) / d(z, S) \leq M$, where $M$ is a constant, then we have

(i)

$$
\begin{aligned}
\lim _{z \rightarrow p} & \frac{1}{\omega} \int_{S} \frac{f(\xi)}{\left(1-z \bar{\xi}^{\prime}\right)^{n+k}} d s(\xi) \\
& =\mathrm{FP} \frac{1}{\omega} \int_{S} \frac{f(\xi)}{\left(1-\bar{\xi}_{1}\right)^{n+k}} d s(\xi)+\frac{1}{2} \frac{1}{(n+k-1) \cdots(n+1) n}\left(L^{k} f\right)(p) .
\end{aligned}
$$

(ii) Let $K=\left(k_{1}, \ldots, k_{n}\right)$ and $k=k_{1}+\cdots+k_{n}$, where $k_{1}, \ldots, k_{n}$ are nonnegative integers. Then

$$
\begin{aligned}
\lim _{z \rightarrow p} & \frac{\partial^{k}}{\partial z_{1}^{k_{1}} \cdots \partial z_{n}^{k_{n}}} \frac{1}{\omega} \int_{S} \frac{f(\xi)}{\left(1-z \bar{\xi}^{\prime}\right)^{n}} d s(\xi) \\
\quad & =\frac{n \cdots(n+k-1)}{\omega} \operatorname{FP} \int_{S} \frac{\left(\bar{\xi}_{1}^{k_{1}} \cdots \bar{\xi}_{n}^{k_{n}}\right) f(\xi)}{\left(1-\bar{\xi}_{1}\right)^{n+k}} d s(\xi)+\left.\frac{1}{2} L^{k}\left[\left(\bar{z}_{1}^{k_{1}} \cdots \bar{z}_{n}^{k_{n}}\right) f(z)\right]\right|_{z=p} .
\end{aligned}
$$

In particular, if $K \neq\left(k_{1}, 0, \ldots, 0\right)$, we have

$$
\left.\frac{1}{2} L^{k}\left[\left(\bar{z}_{1}^{k_{1}} \cdots \bar{z}_{n}^{k_{n}}\right) f(z)\right]\right|_{z=p}=0
$$

and

$$
\begin{aligned}
\lim _{z \rightarrow p} & \frac{\partial^{k}}{\partial z_{1}^{k_{1}} \cdots \partial z_{n}^{k_{n}}} \frac{1}{\omega} \int_{S} \frac{f(\xi)}{\left(1-z \bar{\xi}^{\prime}\right)^{n}} d s(\xi) \\
\quad= & \frac{n \cdots(n+k-1)}{\omega} \mathrm{FP} \int_{S} \frac{\left(\bar{\xi}_{1}^{k_{1}} \cdots \bar{\xi}_{n}^{k_{n}}\right) f(\xi)}{\left(1-\bar{\xi}_{1}\right)^{n+k}} d s(\xi) .
\end{aligned}
$$

Proof. (i) Let $f^{\prime}, f_{1}$ be the same as in the proof of Theorem 2.5. Then, according to Lemma 2.3, we have

$$
\begin{aligned}
& \int_{S} \frac{f(\xi)}{\left(1-z \bar{\xi}^{\prime}\right)^{n+k}} d s(\xi)=\int_{S} \frac{f^{\prime}(\xi)}{\left(1-z \bar{\xi}^{\prime}\right)^{n+k}} d s(\xi)+\int_{S} \frac{f_{1} f(\xi)}{\left(1-z \bar{\xi}^{\prime}\right)^{n+k}} d s(\xi) \\
& \quad=\int_{S} \frac{f^{\prime}(\xi)}{\left(1-z \bar{\xi}^{\prime}\right)^{n+k}} d s(\xi)+\frac{1}{n(n+1) \cdots(n+k-1) z_{1}^{k}} \int_{S} \frac{L^{k}\left(f_{1} f\right)}{\left(1-z \bar{\xi}^{\prime}\right)^{n}} d s(\xi) .
\end{aligned}
$$

According to the proof of Theorem 2.5 we know that

$$
\begin{aligned}
\lim _{z \rightarrow p} & \frac{1}{\omega} \int_{S} \frac{f(\xi)}{\left(1-z \bar{\xi}^{\prime}\right)^{n+k}} d s(\xi)=\frac{1}{\omega} \int_{S} \frac{f^{\prime}(\xi)}{\left(1-\bar{\xi}_{1}\right)^{n+k}} d s(\xi) \\
& +\lim _{z \rightarrow p} \frac{1}{n(n+1) \cdots(n+k-1) \omega} \int_{S} \frac{L^{k}\left(f_{1} f\right)(\xi)}{\left(1-z \bar{\xi}^{\prime}\right)^{n}} d s(\xi) \\
= & \frac{1}{\omega} \int_{S} \frac{f^{\prime}(\xi)}{\left(1-\bar{\xi}_{1}\right)^{n+k}} d s(\xi)+\frac{1}{n(n+1) \cdots(n+k-1) \omega} \mathrm{PV} \int_{S} \frac{L^{k}\left(f_{1} f\right)}{\left(1-\bar{\xi}_{1}\right)^{n}} d s(\xi) \\
& +\frac{1}{2 n(n-1) \cdots(n+k-1)} L^{k}\left(f_{1} f\right)(p) \\
= & \frac{1}{\omega} \mathrm{FP} \int_{S} \frac{f(\xi)}{\left(1-\bar{\xi}_{1}\right)^{n+k}} d s(\xi)+\frac{1}{2 n(n-1) \cdots(n+k-1)} L^{k}(f)(p) .
\end{aligned}
$$


(ii) Since we have the following relation:

$$
\begin{aligned}
& \frac{\partial^{k}}{\partial z_{1}^{k_{1}} \cdots \partial z_{n}^{k_{n}}} \frac{1}{\omega} \int_{S} \frac{f(\xi)}{\left(1-z \bar{\xi}^{\prime}\right)^{n}} d s(\xi) \\
& \quad=\frac{(n+k-1) \cdots n}{\omega} \int_{S} \frac{\left(\bar{\xi}_{1}^{k_{1}} \cdots \bar{\xi}_{n}^{k_{n}}\right) f(\xi)}{\left(1-z \bar{\xi}^{\prime}\right)^{n+k}} d s(\xi),
\end{aligned}
$$

we can deduce (ii) from (i).

We now consider the finite part at an arbitrary point on $S$ by reduction to the case already discussed. For any point $v \in S$, take a unitary matrix $U$ such that $v U=p=(1,0, \ldots, 0)$. Let $\xi=w \bar{U}^{\prime}$. Then we have

$$
\begin{aligned}
& \int_{S \cap\left\{\left|1-v \bar{\xi}^{\prime}\right| \geq \varepsilon\right\}} \frac{f(\xi)}{\left(1-v \bar{\xi}^{\prime}\right)^{n-\beta+k}} d s(\xi) \\
& \quad=\int_{S \cap\left\{\left|1-w_{1}\right| \geq \varepsilon\right\}} \frac{f\left(w \bar{U}^{\prime}\right)}{\left(1-\bar{w}_{1}\right)^{n-\beta+k}} d s(w) .
\end{aligned}
$$

Definition 2.7. The finite part of a singular integral

$$
\int_{S} \frac{f(\xi)}{\left(1-v \bar{\xi}^{\prime}\right)^{n-\beta+k}} d s(\xi)
$$

is defined by

$$
\mathrm{FP} \int_{S} \frac{f(\xi)}{\left(1-v \bar{\xi}^{\prime}\right)^{n-\beta+k}} d s(\xi)=\mathrm{FP} \int_{S} \frac{f\left(w \bar{U}^{\prime}\right)}{\left(1-\bar{w}_{1}\right)^{n-\beta+k}} d s(w) .
$$

The above definition is independent of the choice of the matrix $U$. If $f$ is $k$ times continuously differentiable and the derivatives are Hölder continuous, then the finite part at the point $v \in S$ exists. For points $z$ with $z \bar{z}^{\prime}<1$, write $z U=\eta$. We obtain

$$
\begin{aligned}
\lim _{z \rightarrow v} & \int_{S} \frac{f(\xi)}{\left(1-z \bar{\xi}^{\prime}\right)^{n+k}} d s(\xi)=\lim _{\eta \rightarrow p} \int_{S} \frac{f\left(w \bar{U}^{\prime}\right)}{\left(1-\eta \bar{w}^{\prime}\right)^{n+k}} d s(w) \\
\quad & \operatorname{FP} \int_{S} \frac{f\left(w \bar{U}^{\prime}\right)}{\left(1-\bar{w}_{1}\right)^{n+k}} d s(w)+\left.\frac{\omega}{2(n+k-1) \cdots n} L^{k}\left(f\left(w \bar{U}^{\prime}\right)\right)\right|_{w=p} \\
\quad & \operatorname{FP} \int_{S} \frac{f(\xi)}{\left(1-v \bar{\xi}^{\prime}\right)^{n+k}} d s(\xi)+\left.\frac{\omega}{2(n+k-1) \cdots n} L^{k}\left(f\left(w \bar{U}^{\prime}\right)\right)\right|_{w=p} .
\end{aligned}
$$

\section{FINITE PART OF SINGULAR INTEGRALS OF BOCHNER-MARTINELLI TYPE}

Let $D$ be a domain in $\mathbf{C}^{n} \cong \mathbf{R}^{2 n}$ whose boundary $\partial D=\Omega$ is of class $C^{3}$. Write $z_{k}=u_{k}+i u_{n+k}, k=1, \ldots, n$. We define a Bochner-Martinelli type integral by

$$
F(w)=\int_{\Omega} f(z) K_{2 n-1}(z, w), \quad w \in \mathbf{C}^{n} \backslash \Omega,
$$

where

$$
K_{2 n-1}(z, w)=C_{n} \sum_{j=1}^{n}(-1)^{j-1} \frac{\bar{z}_{j}-\bar{w}_{j}}{|z-w|^{2 n}} d z_{1} \cdots d z_{n} d \bar{z}_{1} \cdots\left[d \bar{z}_{j}\right] \cdots d \bar{z}_{n} .
$$


Here $C_{n}$ is a constant:

$$
C_{n}=\frac{(n-1) !}{(2 \pi i)^{n}} .
$$

$\mathrm{Lu}$ and Zhong [1957] have studied the boundary value of the Bochner-Martinelli type integral and the corresponding Plemelj jump formulas. Their results are the following two lemmas.

Lemma 3.1. Let $f$ be a Hölder-continuous function on $\partial D=\Omega$ and let $a \in \Omega$. Then the principal value

$$
\mathrm{PV} \int_{\Omega} f(z) K_{2 n-1}(z, a)=\lim _{\varepsilon \rightarrow 0} \int_{\Omega \backslash B(a, \varepsilon)} f(z) K_{2 n-1}(z, a)
$$

exists, where $B(a, \varepsilon)=\left\{z \in \mathbf{C}^{n} ;|z-a|<\varepsilon\right\}$.

Lemma 3.2. Let $f$ be as in Lemma 3.1. Then for every $a \in \Omega$ we have

$$
\begin{aligned}
& F_{i}(a)=\mathrm{PV} \int_{\Omega} f(z) K_{2 n-1}(z, a)+\frac{1}{2} f(a), \\
& F_{e}(a)=\mathrm{PV} \int_{\Omega} f(z) K_{2 n-1}(z, a)-\frac{1}{2} f(a),
\end{aligned}
$$

where $F_{i}(a)$ and $F_{e}(a)$ are the limits of $F(w)$ as $w$ tends to $a$ in $D$ and in $\mathrm{C}^{n} \backslash(\Omega \cup D)$, respectively.

Now assume that $0 \in \Omega$ and that in a neighborhood $U$ of the origin we have

$$
\Omega \cap U=\left\{z \in U ; r\left(u_{1}, \ldots, u_{2 n}\right)=0\right\},
$$

for a function $r \in C^{3}(U)$ satisfying

$$
\left.\frac{\partial r}{\partial u_{1}}\right|_{u=0}=1,\left.\quad \frac{\partial r}{\partial u_{2}}\right|_{u=0}=\cdots=\left.\frac{\partial r}{\partial u_{2 n}}\right|_{u=0}=0 .
$$

In other words, the tangent plane of $\Omega$ at the origin is defined by $u_{1}=0$. Since $\partial r /\left.\partial u_{1}\right|_{u=0} \neq 0$, we can write the equation $r\left(u_{1}, \ldots, u_{2 n}\right)=0$ as $u_{1}-h\left(u_{2}, \ldots, u_{2 n}\right)=0$, for a suitable function $h$ of class $C^{3}$ and satisfying $h(0, \ldots, 0)=0, \partial h /\left.\partial u_{2}\right|_{u=0}=\cdots=\partial h /\left.\partial u_{2 n}\right|_{u=0}=0$.

Lemma 3.3. Define $\Omega_{\varepsilon, \eta}=\{z \in \Omega ; \varepsilon<|z|<\eta\}$ for $0<\varepsilon<\eta$. Fix $\eta$. Then

$$
\lim _{\varepsilon \rightarrow 0} \int_{\Omega_{\varepsilon, \eta}} \frac{u_{j} d u_{2} \cdots d u_{2 n}}{|z|^{2 n}}, \quad j=1,2, \ldots, 2 n ;
$$

exists. Moreover, if $w=\left(w_{1}, \ldots, w_{n}\right)=\left(-\varepsilon^{2}, 0, \ldots, 0\right)$, then

(3.2) $\lim _{\varepsilon \rightarrow 0} \int_{\Omega_{\varepsilon, \eta}}\left|\frac{\bar{z}_{j}-\bar{w}_{j}}{|z-w|^{2 n}}-\frac{\bar{z}_{j}}{|z|^{2 n}}\right| d u_{2} \cdots d u_{2 n}=0, \quad j=1,2, \ldots, n$;

(3.3) $\lim _{\varepsilon \rightarrow 0} \int_{\Omega \cap B(0, \varepsilon)} \frac{\bar{z}_{j}-\bar{w}_{j}}{|z-w|^{2 n}} d u_{2} \cdots d u_{2 n}= \begin{cases}\frac{\pi^{n}}{(n-1) !}, & j=1 ; \\ 0, & j=2, \ldots, n \text {; }\end{cases}$

(3.4) $\lim _{\varepsilon \rightarrow 0} \int_{\Omega \cap B(0, \varepsilon)}|z|^{\alpha} \frac{\left|\bar{z}_{j}-\bar{w}_{j}\right|}{|z-w|^{2 n}} d u_{2} \cdots d u_{2 n}=0, \quad j=1,2, \ldots, n$; 


$$
\lim _{\varepsilon \rightarrow 0} \int_{\Omega_{\varepsilon, \eta}}\left|\frac{|z|^{\alpha}\left(\bar{z}_{j}-\bar{w}_{j}\right)}{|z-w|^{2 n}}-\frac{|z|^{\alpha} \bar{z}_{j}}{|z|^{2 n}}\right| d u_{2} \cdots d u_{2 n}=0, \quad j=1,2, \ldots, n .
$$

Here $\alpha$ denotes any positive constant.

Proof. We shall use spherical coordinates

$$
\begin{array}{r}
u_{1}=u_{1}, u_{2}=\rho \cos \theta_{1}, \ldots, u_{2 n-1}=\rho \sin \theta_{1} \cdots \sin \theta_{2 n-3} \cos \theta_{2 n-2}, \\
u_{2 n}=\rho \sin \theta_{1} \cdots \sin \theta_{2 n-3} \sin \theta_{2 n-2} .
\end{array}
$$

Proof of (3.1). We shall only prove the case of $j=2$. The proof for $j=$ $3, \ldots, 2 n$ is similar. For $j=1$, the function is even integrable.

By using spherical coordinates we have

$$
\begin{gathered}
\int_{\Omega_{\varepsilon, \eta}} \frac{u_{2} d u_{2} \cdots d u_{2 n}}{|z|^{2 n}}=\int_{0}^{\pi} \sin ^{2 n-3} \theta_{1} \cos \theta_{1} d \theta_{1} \int_{0}^{\pi} \sin ^{2 n-4} \theta_{2} d \theta_{2} \\
\quad \ldots \int_{0}^{\pi} \sin \theta_{2 n-3} d \theta_{2 n-3} \int_{0}^{2 \pi} d \theta_{2 n-2} \int_{\varepsilon^{2}<\psi^{2}+\rho^{2}<\eta^{2}} \frac{\rho^{2 n-1} d \rho}{\left(\rho^{2}+\psi(\rho, \theta)^{2}\right)^{n}}
\end{gathered}
$$

where $\psi(\rho, \theta)=h\left(\rho \cos \theta_{1}, \ldots, \rho \sin \theta_{1} \ldots \sin \theta_{2 n-2}\right)$.

It is clear that

$$
\psi(0, \theta)=0, \quad \frac{\partial \psi}{\partial \rho}(0, \theta)=0 ;
$$

we can therefore assume $\psi(\rho, \theta)=\rho^{2} \varphi(\rho, \theta)$ for some $\varphi(\rho, \theta)$ which is of class $C^{3}$ outside $\rho=0$ and continuous at $\rho=0$.

Consider

$$
g(\theta)=\int_{\varepsilon^{2}<\psi^{2}+\rho^{2}<\eta^{2}} \frac{\rho^{2 n-1} d \rho}{\left(\rho^{2}+\psi(\rho, \theta)^{2}\right)^{n}} .
$$

Let $t=\sqrt{\rho^{2}+\psi^{2}}$. Then

$$
\psi(\rho, \theta)=\rho^{2} \varphi(\rho, \theta)=t^{2} \varphi_{1}(t, \theta),
$$

where $\varphi_{1}(t, \theta)$ is $C^{3}$ outside $t=0$ and continuous at $t=0$. We get

$$
\begin{aligned}
g(\theta) & =\int_{\varepsilon}^{\eta} \frac{\left(t^{2}-\psi^{2}\right)^{n-1}}{t^{2 n-1}} d t-\int_{\varepsilon}^{\eta} \frac{\left(t^{2}-\psi^{2}\right)^{n-1}}{t^{2 n}} \psi \frac{\partial \psi}{\partial t} d t \\
& =\int_{\varepsilon}^{\eta} \frac{\left(1-t^{2} \varphi_{1}^{2}\right)^{n-1}}{t} d t-\int_{\varepsilon}^{\eta}\left(1-t^{2} \varphi_{1}^{2}\right)^{n-1} \varphi_{1} \frac{\partial \psi}{\partial t} d t \\
& =\int_{\varepsilon}^{\eta} \frac{1}{t} d t+\int_{\varepsilon}^{\eta} \sum_{p=1}^{n-1}(-1)^{p} C_{n-1}^{p} t^{2 p-1} \varphi_{1}^{2 p} d t-\int_{\varepsilon}^{\eta}\left(1-t^{2} \varphi_{1}^{2}\right)^{n-1} \varphi_{1} \frac{\partial \psi}{\partial t} d t \\
& =\log \eta-\log \varepsilon+\int_{\varepsilon}^{\eta} \sum_{p=1}^{n-1}(-1)^{p} C_{n-1}^{p} t^{2 p-1} \varphi_{1}^{2 p} d t-\int_{\varepsilon}^{\eta}\left(1-t^{2} \varphi_{1}^{2}\right)^{n-1} \varphi_{1} \frac{\partial \psi}{\partial t} d t,
\end{aligned}
$$

$C_{n-1}^{p}=(n-1) ! /(p !(n-p-1) !)$ denoting the binomial coefficients. 
Using the known result that $\int_{0}^{\pi} \sin ^{2 n-3} \theta_{1} \cos \theta_{1} d \theta_{1}=0$, we get

$$
\begin{aligned}
\int_{\Omega_{\varepsilon, \eta}} & \frac{u_{2} d u_{2} \cdots d u_{2 n}}{|z|^{2}} \\
= & \int_{0}^{\pi} \sin ^{2 n-3} \theta_{1} \cos \theta_{1} d \theta_{1} \int_{0}^{\pi} \sin ^{2 n-4} \theta_{2} d \theta_{2} \ldots \\
& \int_{0}^{\pi} \sin \theta_{2 n-3} d \theta_{2 n-3} \int_{0}^{2 \pi} g(\theta) d \theta_{2 n-3} \\
= & \int_{0}^{\pi} \sin ^{2 n-3} \theta_{1} \cos \theta_{1} d \theta_{1} \ldots \\
\int_{\varepsilon}^{2 \pi} \sum_{p=1}^{n-1}(-1)^{p} C_{n-1}^{p} t^{2 p-1} \varphi_{1}^{2 p} d t & \left.-\int_{\varepsilon}^{\eta}\left(1-t^{2} \varphi_{1}^{2}\right)^{n-1} \varphi_{1} \frac{\partial \psi}{\partial t} d t\right\} d \theta_{2 n-2} .
\end{aligned}
$$

The integrals in braces are continuous functions of $\varepsilon$. So when $\varepsilon \rightarrow 0$, the limit exists.

Proof of (3.2). Applying spherical coordinates we know that

$$
\begin{aligned}
\int_{\Omega_{\varepsilon, \eta}}\left|\frac{u_{2}}{|z-w|^{2 n}}-\frac{u_{2}}{|z|^{2 n}}\right| d u_{2} \cdots d u_{2 n} \\
=\int_{0}^{\pi} \sin ^{2 n-3} \theta_{1}\left|\cos \theta_{1}\right| d \theta_{1} \int_{0}^{\pi} \sin ^{2 n-4} \theta_{2} d \theta_{2} \cdots \\
\int_{0}^{\pi} \sin \theta_{2 n-3} d \theta_{2 n-3} \int_{0}^{2 \pi} d \theta_{2 n-2} \\
\cdot \int_{\varepsilon}^{\eta}\left[\frac{1}{\left[t^{2}+\varepsilon^{4}+2 \varepsilon^{2} t^{2} \varphi_{1}(t, \eta)\right]^{n}}-\frac{1}{t^{2 n}}\right] t^{2 n-1} \\
\cdot\left[\left(1-t^{2} \varphi_{1}^{2}\right)^{n-1}-\left(1-t^{2} \varphi_{1}^{2}\right)^{n-1} t \varphi_{1} \frac{\partial \psi}{\partial t}\right] d t
\end{aligned}
$$

Notice that $\left(\varepsilon^{4}+2 \varepsilon^{2} t^{2} \varphi_{1}\right) / t^{2}=O\left(\varepsilon^{2}\right), t \in[\varepsilon, \eta]$, and that

$$
\begin{aligned}
& \frac{1}{\left[t^{2}+\varepsilon^{4}+2 \varepsilon^{2} t^{2} \varphi_{1}(t, \eta)\right]^{n}} \\
& \quad=\frac{1}{t^{2 n}\left(1+\left(\varepsilon^{4}+2 \varepsilon^{2} t^{2} \varphi_{1}\right) / t^{2}\right)^{n}}=\frac{1}{t^{2 n}}+O\left(\frac{\varepsilon^{2}}{t^{2 n}}\right) .
\end{aligned}
$$

Applying the above estimate to (3.7), we obtain

$$
\int_{\Omega_{\varepsilon, \eta}}\left|\frac{u_{2}}{|z-w|^{2 n}}-\frac{u_{2}}{|z|^{2 n}}\right| d u_{2} \cdots d u_{2 n} \leq C \varepsilon^{2} \int_{\varepsilon}^{\eta} \frac{1}{t} d t
$$

Similarly we can prove that

$$
\int_{\Omega_{\varepsilon, \eta}}\left|\frac{u_{j}}{|z-w|^{2 n}}-\frac{u_{j}}{|z|^{2 n}}\right| d u_{2} \cdots d u_{2 n} \leq C \varepsilon^{2} \int_{\varepsilon}^{\eta} \frac{1}{t} d t, \quad j=3, \ldots, 2 n,
$$


which implies (3.2) for the case $j=2, \ldots, n$. Since $u_{1}=o\left(\left|u_{2}\right|+\cdots+\left|u_{2 n}\right|\right)$, on $\Omega_{\varepsilon, \eta}$, we see that

$$
\lim _{\varepsilon \rightarrow 0} \int_{\Omega_{\varepsilon, \eta}}\left|\frac{u_{1}}{|z-w|^{2 n}}-\frac{u_{1}}{|z|^{2 n}}\right| d u_{2} \cdots d u_{2 n}=0 .
$$

On the other hand we have the following estimates

$$
\int_{\Omega_{\varepsilon, \eta}} \frac{\varepsilon^{2}}{|z-w|^{2 n}} d u_{2} \cdots d u_{2 n} \leq C \varepsilon^{2} \int_{\varepsilon}^{\eta} \frac{1}{t^{2}} d t
$$

Therefore we obtain (3.2) when $j=1$.

Proof of (3.3). Applying the spherical coordinates (3.6) we know that

$$
\begin{gathered}
\int_{\Omega \cap B(0, \varepsilon)} \frac{u_{2}}{|z-w|^{2 n}} d u_{2} \cdots d u_{2 n} \\
=\int_{0}^{\pi} \sin ^{2 n-3} \theta_{1} \cos \theta_{1} d \theta_{1} \int_{0}^{\pi} \sin ^{2 n-4} \theta_{2} d \theta_{2} \cdots \\
\cdot \int_{0}^{\varepsilon} \frac{1}{\sin \theta_{2 n-3} d \theta_{2 n-3} \int_{0}^{2 \pi} d \theta_{2 n-2}} \\
\cdot\left[\left(1-t^{2} \varphi_{1}^{2}\right)^{n-1}-\left(1-t^{2} \varphi_{1}^{2}\right)^{n-1} t \varphi_{1} \frac{\partial \psi}{\partial t}\right] d t
\end{gathered}
$$

Notice that for $t \in[0, \varepsilon], 2 \varepsilon^{2} t^{2} \varphi_{1}(t, \theta) /\left(t^{2}+\varepsilon^{4}\right)=O\left(\varepsilon^{2}\right)$. So we have

$$
\frac{1}{t^{2}+\varepsilon^{4}+2 \varepsilon^{2} t^{2} \varphi_{1}(t, \eta)}=\frac{1}{\left(t^{2}+\varepsilon^{4}\right)}\left(1+O\left(\varepsilon^{2}\right)\right) \text {. }
$$

Applying the above estimate and $\int_{0}^{\pi} \sin ^{2 n-3} \theta_{1} \cos \theta_{1} d \theta_{1}=0$ to (3.8), we obtain

$$
\begin{gathered}
\left|\int_{\Omega \cap B(0, \varepsilon)} \frac{u_{2}}{|z-w|^{2 n}} d u_{2} \cdots d u_{2 n}\right| \leq C \varepsilon^{2} \int_{0}^{\varepsilon} \frac{t^{2 n-1}}{\left(t^{2}+\varepsilon^{4}\right)^{n}} d t \\
\leq C \varepsilon^{2} \int_{0}^{\varepsilon} \frac{t}{t^{2}+\varepsilon^{4}} d t=\frac{C \varepsilon^{2}}{2} \int_{0}^{\varepsilon} \frac{d\left(t^{2}\right)}{t^{2}+\varepsilon^{4}} \\
\quad=\frac{C \varepsilon^{2}}{2} \log \left(1+1 / \varepsilon^{2}\right) \rightarrow 0 .
\end{gathered}
$$

Similarly we can prove that

$$
\left|\int_{\Omega \cap B(0, \varepsilon)} \frac{u_{j}}{|z-w|^{2 n}} d u_{2} \cdots d u_{2 n}\right| \leq C \varepsilon^{2} \int_{0}^{\varepsilon} \frac{d t^{2}}{t^{2}+\varepsilon^{4}}, \quad j=2, \ldots, 2 n,
$$

which implies (3.3) when $j=2, \ldots, n$.

Since $\partial h / \partial u_{j}(0)=0$ for $j=2, \ldots, 2 n$ we see that

$$
\left|u_{1}\right| \leq C\left(\left|u_{2}\right|^{2}+\cdots+\left|u_{n}\right|^{2}\right)
$$


on $\Omega_{0, \eta}$. So we obtain the inequality

$$
\begin{aligned}
& \left|\int_{\Omega \cap B(0, \varepsilon)} \frac{u_{1}}{|z-w|^{2 n}} d u_{2} \cdots d u_{2 n}\right| \\
& \quad \leq\left|\int_{\Omega \cap B(0, \varepsilon)} \frac{|z|^{2}}{|z-w|^{2 n}} d u_{2} \cdots d u_{2 n}\right| \leq C \int_{0}^{\varepsilon} \frac{t^{2 n}}{\left(t^{2}+\varepsilon^{4}\right)^{n}} d t,
\end{aligned}
$$

where the last inequality is obtained as in the proof of (3.2) when $j=1$. Similarly we see that

$$
\begin{aligned}
& \int_{\Omega \cap B(0, \varepsilon)} \frac{\varepsilon^{2}}{|z-w|^{2 n}} d u_{2} \cdots d u_{2 n} \\
& \quad=\int_{0}^{\pi} \sin ^{2 n-3} \theta_{1} d \theta_{1} \cdots \int_{0}^{\pi} \sin \theta_{2 n-3} d \theta_{2 n-3} \int_{0}^{2 \pi} d \theta_{2 n-2} \int_{0}^{\varepsilon} \frac{\varepsilon^{2} t^{2 n-2}}{\left(t^{2}+\varepsilon^{4}\right)^{n}} d t+o(1) \\
& \quad=\int_{0}^{\pi} \sin ^{2 n-3} \theta_{1} d \theta_{1} \cdots \int_{0}^{\pi} \sin \theta_{2 n-3} d \theta_{2 n-3} \int_{0}^{2 \pi} d \theta_{2 n-2} \int_{0}^{\infty} \frac{t^{2 n-2}}{\left(t^{2}+1\right)^{n}} d t+o(1) \\
& =(2 n-1) b_{2 n-1} \int_{0}^{\infty} \frac{t^{2 n-2}}{\left(t^{2}+1\right)^{n}} d t+o(1) .
\end{aligned}
$$

Here

$$
b_{2 n-1}=\frac{2^{n} \pi^{n-1}}{(2 n-1) ! !}
$$

is the volume of the unit ball in $\mathbf{R}^{2 n-1}$ and the value of the integral

$$
I_{n}=\int_{0}^{\infty} \frac{t^{2 n-2}}{\left(t^{2}+1\right)^{n}} d t
$$

is easily found to be

$$
I_{n}=\frac{\pi}{2} \frac{(2 n-3) ! !}{(2 n-2) ! !}=\frac{\pi(2 n-3) ! !}{2^{n}(n-1) !}
$$

so that the last expression becomes $\pi^{n} /(n-1) !+o(1)$. Combining with the above estimates we get that

$$
\begin{aligned}
& \lim _{\varepsilon \rightarrow 0} \int_{\Omega \cap B(0, \varepsilon)} \frac{\bar{z}_{1}-\bar{w}_{1}}{|z-w|^{2 n}} d u_{2} \cdots d u_{2 n} \\
& \quad=(2 n-1) b_{2 n-1} \int_{0}^{\infty} \frac{t^{2 n-2}}{\left(t^{2}+1\right)^{n}} d t=\frac{\pi^{n}}{(n-1) !} .
\end{aligned}
$$

Proof of (3.4). Similarly as in the previous step we can prove the estimates

$$
\int_{\Omega \cap B(0, \varepsilon)}|z|^{\alpha} \frac{\bar{z}_{j}-\bar{w}_{j}}{|z-w|^{2 n}} d u_{2} \cdots d u_{2 n} \leq \begin{cases}C \varepsilon^{2} \int_{0}^{\varepsilon} \frac{t^{2 n+\alpha-2}}{\left(t^{2}+\varepsilon^{4}\right)^{n}} d t, & j=1, \\ C \int_{0}^{\varepsilon} \frac{t^{2 n+\alpha-1}}{\left(t^{2}+\varepsilon^{4}\right)^{n}} d t, & j=2, \ldots, n,\end{cases}
$$

which is what we have to prove. 
Proof of (3.5). As in the proof of (3.2) we get

$$
\int_{\Omega_{\varepsilon, \eta}}\left|\frac{|z|^{\alpha}\left(\bar{z}_{j}-\bar{w}_{j}\right)}{|z-w|^{2 n}}-\frac{|z|^{\alpha} \bar{z}_{j}}{|z|^{2 n}}\right| d u_{2} \cdots d u_{2 n} \leq \varepsilon^{2} \int_{\varepsilon}^{\eta} \frac{t^{\alpha}}{t^{2}} d t
$$

which is what we want.

Theorem 3.4. Suppose $\partial h / \partial u_{i}, i=2, \ldots, 2 n$, are Hölder continuous on $\Omega$ and $f$ is a continuously differentiable function on $\Omega$ such that $\partial f / \partial u_{j}, j=$ $1, \ldots, 2 n$, are Hölder continuous. Then the finite part

$$
\mathrm{FP} \int_{\Omega} f(z) \frac{\bar{z}_{k}}{|z|^{2}} K_{2 n-1}(z, 0)
$$

defined as

exists, where

$$
\lim _{\varepsilon \rightarrow 0}\left[\int_{\Omega \backslash B(0, \varepsilon)} f(z) \frac{\bar{z}_{k}}{|z|^{2}} K_{2 n-1}(z, 0)-\frac{B_{k} f(0)}{\varepsilon}\right],
$$

$$
B_{k}= \begin{cases}2^{n-1} i^{n} C_{n} b_{2 n-1}=\frac{2^{n-1}(n-1) !}{\pi(2 n-1) ! !}, & k=1 ; \\ 0, & k=2, \ldots, n ;\end{cases}
$$

here $b_{2 n-1}$ is the volume of the unit ball of dimension $2 n-1$ and $C_{n}$ is the constant in the Bochner-Martinelli kernel.

Proof. We prove only the case of $k=1$; other cases can be proved similarly. By Stokes' formula we get

$$
\begin{aligned}
\int_{\Omega \backslash B(0, \varepsilon)} & f(z) \frac{\bar{z}_{1}}{|z|^{2 n+2}} \sum_{j=1}^{n}(-1)^{j-1} \bar{z}_{j} d z_{1} \cdots d z_{n} d \bar{z}_{1} \cdots\left[d \bar{z}_{j}\right] \cdots d \bar{z}_{n} \\
= & -\frac{1}{n} \int_{\Omega \backslash B(0, \varepsilon)} f(z) d\left[\frac{\sum_{j=1}^{n}(-1)^{j-1} \bar{z}_{j} d z_{2} \cdots d z_{n} d \bar{z}_{1} \cdots\left[d \bar{z}_{j}\right] \cdots d \bar{z}_{n}}{|z|^{2 n}}\right] \\
= & -\frac{1}{n} \int_{\partial(\Omega \cap B(0, \varepsilon))} f(z) \frac{\sum_{j=1}^{n}(-1)^{j-1} \bar{z}_{j} d z_{2} \cdots d z_{n} d \bar{z}_{1} \cdots\left[d \bar{z}_{j}\right] \cdots d \bar{z}_{n}}{|z|^{2 n}} \\
& +\frac{1}{n} \int_{\Omega \backslash B(0, \varepsilon)} d f(z) \frac{\sum_{j=1}^{n}(-1)^{j-1} \bar{z}_{j} d z_{2} \cdots d z_{n} d \bar{z}_{1} \cdots\left[d \bar{z}_{j}\right] \cdots d \bar{z}_{n}}{|z|^{2 n}}
\end{aligned}
$$

Let

$$
\psi(\varepsilon)=-\frac{C_{n}}{n \varepsilon^{2 n}} \int_{\partial(B(0, \varepsilon) \cap \Omega)} f(z) \sum_{j=1}^{n}(-1)^{j-1} \bar{z}_{j} d z_{2} \cdots d z_{n} d \bar{z}_{1} \cdots\left[d \bar{z}_{j}\right] \cdots d \bar{z}_{n} .
$$

Then

$$
\begin{aligned}
& \int_{\Omega \backslash B(0, \varepsilon)} f(z) \frac{\bar{z}_{1}}{|z|^{2}} K_{2 n-1}(z, 0)-\psi(\varepsilon) \\
& =\frac{1}{n} \int_{\Omega \backslash B(0, \varepsilon)} \frac{\partial f}{\partial z_{1}} K_{2 n-1}(z, 0) \\
& \quad+\frac{C_{n}}{n}(-1)^{n-1} \int_{\Omega \backslash B(0, \varepsilon)} \frac{1}{|z|^{2 n}} \sum_{j=1}^{n} \frac{\partial f}{\partial \bar{z}_{j}} \bar{z}_{j} d z_{2} \cdots d z_{n} d \bar{z}_{1} \cdots d \bar{z}_{n} \\
& =I_{1}+I_{2}
\end{aligned}
$$


According to Lemma 3.1, $\lim _{\varepsilon \rightarrow 0} I_{1}$ exists.

$$
\begin{aligned}
I_{2} & =\frac{C_{n}}{n}(-1)^{n-1}\left\{\int_{\Omega \backslash B(0, \eta)}+\int_{\Omega_{\varepsilon, \eta}}\right\} \frac{1}{|z|^{2 n}} \sum_{j=1}^{n} \frac{\partial f}{\partial \bar{z}_{j}} \bar{z}_{j} d z_{2} \cdots d z_{n} d \bar{z}_{1} \cdots d \bar{z}_{n} \\
& =I_{1}^{\prime}+I_{1}^{\prime} .
\end{aligned}
$$

If $\eta$ is small enough, $\lim _{\varepsilon \rightarrow 0} I_{1}^{\prime}$ exists because $I_{1}^{\prime}$ is independent of $\varepsilon$.

$$
I_{2}^{\prime}=\frac{(-2 i)^{n-1} C_{n}}{n} \int_{\Omega_{\varepsilon, \eta}} \frac{1}{|z|^{2 n}} \sum_{j=1}^{n} \frac{\partial f}{\partial \bar{z}_{j}} \bar{z}_{j} d \bar{z}_{1} d u_{2} \cdots d u_{n} d u_{n+2} \cdots d u_{2 n} .
$$

Notice that

$$
\begin{aligned}
d \bar{z}_{1}= & d u_{1}-i d u_{n+1}=\left(\frac{\partial h}{\partial u_{n+1}}-i\right) d u_{n+1}\left(\bmod d u_{2} \cdots d u_{n} d u_{n+2} \cdots d u_{2 n}\right) \\
I_{2}^{\prime}= & \frac{(-1)^{n-1}(-2 i)^{n-1} C_{n}}{n} \int_{\Omega_{\varepsilon, \eta}} \frac{1}{|z|^{2 n}}\left(\sum_{j=1}^{n} \frac{\partial f}{\partial \bar{z}_{j}} \bar{z}_{j}\right)\left(\frac{\partial h}{\partial u_{n+1}}-i\right) d u_{2} \cdots d u_{2 n} \\
= & \frac{(-1)^{n-1}(-2 i)^{n-1} C_{n}}{n} \int_{\Omega_{\varepsilon, \eta}} \frac{1}{|z|^{2 n}} \\
& \cdot \sum_{j=1}^{n}\left[\frac{\partial f}{\partial \bar{z}_{j}}(z)\left(\frac{\partial h}{\partial u_{n+1}}-i\right)-\left(\frac{\partial f}{\partial \bar{z}_{j}}(0)\right)\left(\frac{\partial h}{\partial u_{n+1}}(0)-i\right)\right] \bar{z}_{j} d u_{2} \cdots d u_{2 n} \\
& +\frac{1}{n}(-1)^{n-1}(-2 i)^{n-1} C_{n} \sum_{j=1}^{n} \frac{\partial f}{\partial \bar{z}_{j}}(0)\left(\frac{\partial h(0)}{\partial u_{n+1}}-i\right) \int_{\Omega_{\varepsilon, \eta}} \frac{1}{|z|^{2 n}} \bar{z}_{j} d u_{2} \cdots d u_{2 n} .
\end{aligned}
$$

Because $\partial f / \partial \bar{z}_{j}$ and $\partial h / \partial u_{n+1}$ are Hölder continuous on $\Omega$, the limit of the first term exists when $\varepsilon \rightarrow 0$. The limit of last term exists by (3.1) in Lemma 3.3. On the other hand, by assumption $\partial f / \partial u_{j}, j=1,2, \ldots, 2 n$, are Hölder continuous, so we have the following identity:

$$
\begin{aligned}
\psi(\varepsilon)= & -\frac{C_{n}}{n \varepsilon^{2 n}} \int_{\partial(B(0, \varepsilon) \cap \Omega)} f(z) \sum_{k=1}^{n}(-1)^{k-1} \bar{z}_{k} d z_{2} \cdots d z_{n} d \bar{z}_{1} \cdots\left[d \bar{z}_{k}\right] \cdots d \bar{z}_{n} \\
= & -\frac{C_{n}}{n \varepsilon^{2 n}} \int_{\partial(B(0, \varepsilon) \cap \Omega)}\left[f(0)+\sum_{j=1}^{n}\left(\frac{\partial f}{\partial z_{j}}(0) z_{j}+\frac{\partial f}{\partial \bar{z}_{j}}(0) \bar{z}_{j}\right)\right] \\
& \cdot \sum_{k=1}^{n}(-1)^{k-1} \bar{z}_{k} d z_{2} \cdots d z_{n} d \bar{z}_{1} \cdots\left[d \bar{z}_{k}\right] \cdots d \bar{z}_{n}+o(1) \\
= & \frac{(-1)^{n} C_{n} f(0)}{\varepsilon^{2 n}} \int_{B(0, \varepsilon) \cap \Omega} d z_{2} \cdots d z_{n} d \bar{z}_{1} \cdots d \bar{z}_{n}+o(1) \\
& -\frac{C_{n}}{n \varepsilon^{2 n}} \frac{\partial f}{\partial z_{1}}(0) \sum_{k=1}^{n}(-1)^{k-1} \int_{B(0, \varepsilon) \cap \Omega} \bar{z}_{k} d z_{1} \cdots d z_{n} d \bar{z}_{1} \cdots\left[d \bar{z}_{k}\right] \cdots d \bar{z}_{n} \\
& +\frac{(-1)^{n} C_{n}}{\varepsilon^{2 n}} \sum_{j=1}^{n} \frac{\partial f}{\partial z_{j}}(0) \int_{B(0, \varepsilon) \cap \Omega} z_{j} d z_{2} \cdots d z_{n} d \bar{z}_{1} \cdots d \bar{z}_{n} \\
& +\frac{(-1)^{n} C_{n}(n+1)}{n \varepsilon^{2 n}} \sum_{j=1}^{n} \frac{\partial f}{\partial \bar{z}_{j}}(0) \int_{B(0, \varepsilon) \cap \Omega} \bar{z}_{j} d z_{2} \cdots d z_{n} d \bar{z}_{1} \cdots d \bar{z}_{n} .
\end{aligned}
$$


The last identity is obtained by Stokes' formula.

By the method used in proving Lemma 3.3 we can prove that the last three terms tend to zero as $\varepsilon$ tends to zero.

$$
\begin{aligned}
& \int_{B(0, \varepsilon) \cap \Omega} d z_{2} \cdots d z_{n} d \bar{z}_{1} \cdots d \bar{z}_{n} \\
&=\int_{B(0, \varepsilon) \cap \Omega}(-1)^{n-1}\left(d u_{1}-i d u_{n+1}\right)(-2 i)^{n-1} d u_{2} \cdots d u_{n} d u_{n+2} \cdots d u_{2 n} \\
&=(-2 i)^{n-1}(-i) \int_{B(0, \varepsilon) \cap \Omega} d u_{2} d u_{3} \cdots d u_{2 n}+(-2 i)^{n-1} \\
& \cdot \int_{B(0, \varepsilon) \cap \Omega} \frac{\partial h}{\partial u_{n+1}} d u_{2} d u_{3} \cdots d u_{2 n} \\
&=(-2 i)^{n-1}(-i) \int_{B(0, \varepsilon) \cap \Omega} d u_{2} d u_{3} \cdots d u_{2 n}+o\left(\varepsilon^{2 n}\right) \\
&=(-2 i)^{n-1}(-i) \int_{0}^{\pi} \sin ^{2 n-3} \theta_{1} d \theta_{1} \cdots \\
& \int_{0}^{\pi} \sin \theta_{2 n-3} d \theta_{2 n-3} \int_{0}^{2 \pi} d \theta_{2 n-2} \int_{\psi^{2}+\rho^{2} \leq \varepsilon^{2}} \rho^{2 n-2} d \rho+o\left(\varepsilon^{2 n}\right) \\
&=(-2 i)^{n-1}(-i) \int_{0}^{\pi} \sin ^{2 n-3} \theta_{1} d \theta_{1} \cdots \\
& \int_{0}^{\pi} \sin \theta_{2 n-3} d \theta_{2 n-3} \int_{0}^{2 \pi} d \theta_{2 n-2} \int_{0}^{\varepsilon} t^{2 n-2} d t+o\left(\varepsilon^{2 n}\right) .
\end{aligned}
$$

Therefore we see that

$$
\psi(\varepsilon)=\frac{i}{\varepsilon}(2 i)^{n-1} C_{n} b_{2 n-1} f(0)+o(1) .
$$

The proof is finished.

Theorem 3.5. Let $f$ and $D$ be as in Theorem 3.4. Define

$$
F(w)=\int_{\Omega} f(z) K(z, w) .
$$

Then the limits of $\left(\partial F / \partial w_{k}\right)(w), k=1, \ldots, n$, as $w$ tends to 0 along the inner normal and the exterior normal of $\Omega$ at the point 0 , exist and are given by the following expressions:

$$
\begin{aligned}
& \left(\frac{\partial F}{\partial w_{k}}\right)_{i}(0)=\operatorname{FP} \int_{\Omega} n f(z) \frac{\bar{z}_{k}}{|z|^{2}} K(z, 0)+\frac{1}{2} \frac{\partial f}{\partial z_{k}}(0)+\frac{1}{2} A_{k} \frac{\partial f}{\partial \bar{z}_{k}}(0) ; \\
& \left(\frac{\partial F}{\partial w_{k}}\right)_{\varepsilon}(0)=\mathrm{FP} \int_{\Omega} n f(z) \frac{\bar{z}_{k}}{|z|^{2}} K(z, 0)-\frac{1}{2} \frac{\partial f}{\partial z_{k}}(0)-\frac{1}{2} A_{k} \frac{\partial f}{\partial \bar{z}_{k}}(0),
\end{aligned}
$$

where

$$
A_{k}= \begin{cases}-(2 i)^{n}(2 n-1) C_{n} b_{2 n-1} \int_{0}^{\infty} \frac{t^{2 n-2} d t}{\left(t^{2}+1\right)^{n}}=-1 ; & k=1, \\ 0 ; & k=2, \ldots, n .\end{cases}
$$

Here $b_{2 n-1}$ is the volume of the unit ball of dimension $2 n-1$ and $C_{n}$ is the constant in the Bochner-Martinelli kernel. 
Proof. We only prove the first equality for the case $k=1$. For the other cases the proof is similar.

$$
\begin{aligned}
\frac{\partial F}{\partial w_{1}}(w)=n C_{n} \int_{\Omega} f(z) \frac{\bar{z}_{1}-\bar{w}_{1}}{|z-w|^{2 n+2}} \sum_{j=1}^{n}(-1)^{j-1}\left(\bar{z}_{j}-\bar{w}_{j}\right) d z_{1} \cdots & d z_{n} d \bar{z}_{1} \cdots\left[d \bar{z}_{j}\right] \cdots d \bar{z}_{n} \\
= & -C_{n} \int_{\Omega} f(z) d\left[\frac{1}{|z-w|^{2 n}} \sum_{j=1}^{n}(-1)^{j-1}\left(\bar{z}_{j}-\bar{w}_{j}\right) d z_{2} \cdots\right. \\
= & C_{n} \int_{\Omega} \frac{\partial f(z)}{\partial z_{1}} \frac{1}{|z-w|^{2 n}} \sum_{j=1}^{n}(-1)^{j-1}\left(\bar{z}_{j}-\bar{w}_{j}\right) d z_{1} \cdots \\
& \left.\left.+(-1)^{n-1} C_{n} \int_{\Omega} \sum_{j=1}^{n} \frac{\partial f}{\partial \bar{z}_{j}} \frac{1}{|z-w|^{2 n}}\left(\bar{z}_{j}-\bar{w}_{j}\right) d z_{2} \cdots d \bar{z}_{j}\right] \cdots d \bar{z}_{n}\right] \\
& \quad \int_{\Omega} \frac{\partial f}{\partial z_{1}} K(z, w) d \bar{z}_{1} \cdots\left[d \bar{z}_{j}\right] \cdots d \bar{z}_{n} \\
& +(-1)^{n-1} C_{n} \int_{\Omega} \frac{1}{|z-w|^{2 n}} \sum_{j=1}^{n} \frac{\partial f}{\partial \bar{z}_{j}}\left(\bar{z}_{j}-\bar{w}_{j}\right) d z_{2} \cdots d z_{n} d \bar{z}_{1} \cdots d \bar{z}_{n} .
\end{aligned}
$$

By the proof of Theorem 3.4 we know that

$$
\begin{aligned}
& \mathrm{FP} \int_{\Omega} f(z) \frac{\bar{z}_{1}}{|z|^{2}} K(z, 0)=\lim _{\varepsilon \rightarrow 0} \frac{1}{n} \int_{\Omega \backslash B(0, \varepsilon)} \frac{\partial f}{\partial z_{1}} K(z, 0) \\
& \quad+\lim _{\varepsilon \rightarrow 0} \frac{C_{n}}{n}(-1)^{n-1} \int_{\Omega \backslash B(0, \varepsilon)} \frac{1}{|z|^{2 n}} \sum_{j=1}^{n} \frac{\partial f}{\partial \bar{z}_{j}} \bar{z}_{j} d z_{2} \cdots d z_{n} d \bar{z}_{1} \cdots d \bar{z}_{n} .
\end{aligned}
$$

So we only need to prove the following inequality

$$
\begin{aligned}
\lim _{w \rightarrow 0} \int_{\Omega} & \frac{\partial f}{\partial z_{1}} K(z, w) \\
& +\lim _{w \rightarrow 0}(-1)^{n-1} C_{n} \int_{\Omega} \frac{1}{|z-w|^{2 n}} \sum_{j=1}^{n} \frac{\partial f}{\partial \bar{z}_{j}}\left(\bar{z}_{j}-\bar{w}_{j}\right) d z_{2} \cdots d z_{n} d \bar{z}_{1} \cdots d \bar{z}_{n} \\
= & \lim _{\varepsilon \rightarrow 0} \int_{\Omega \backslash B(0, \varepsilon)} \frac{\partial f}{\partial z_{1}} K(z, 0)+\frac{1}{2} \frac{\partial f}{\partial z_{1}}(0) \\
& +\lim _{\varepsilon \rightarrow 0} C_{n}(-1)^{n-1} \int_{\Omega \backslash B(0, \varepsilon)} \frac{1}{|z|^{2 n}} \sum_{j=1}^{n} \frac{\partial f}{\partial \bar{z}_{j}} \bar{z}_{j} d z_{2} \cdots d z_{n} d \bar{z}_{1} \cdots d \bar{z}_{n} \\
& +\frac{1}{2} A_{1} \frac{\partial f}{\partial \bar{z}_{1}}(0) .
\end{aligned}
$$


It follows from Lemma 3.2 that

$$
\lim _{w \rightarrow 0} \int_{\Omega} \frac{\partial f}{\partial z_{1}} K(z, w)=\lim _{\varepsilon \rightarrow 0} \int_{\Omega \backslash B(0, \varepsilon)} \frac{\partial f}{\partial z_{1}} K(z, 0)+\frac{1}{2} \frac{\partial f}{\partial z_{1}}(0) .
$$

So we only need to prove that

$$
\begin{aligned}
\lim _{w \rightarrow 0}( & -1)^{n-1} C_{n} \int_{\Omega} \frac{1}{|z-w|^{2 n}} \sum_{j=1}^{n} \frac{\partial f}{\partial \bar{z}_{j}}\left(\bar{z}_{j}-\bar{w}_{j}\right) d z_{2} \cdots d z_{n} d \bar{z}_{1} \cdots d \bar{z}_{n} \\
= & \lim _{\varepsilon \rightarrow 0}(-1)^{n-1} C_{n} \int_{\Omega \backslash B(0, \varepsilon)} \frac{1}{|z|^{2 n}} \sum_{j=1}^{n} \frac{\partial f}{\partial \bar{z}_{j}} \bar{z}_{j} d z_{2} \cdots d z_{n} d \bar{z}_{1} \cdots d \bar{z}_{n} \\
& +\frac{1}{2} A_{1} \frac{\partial f}{\partial \bar{z}_{1}}(0) .
\end{aligned}
$$

Take $w$ on the inner normal. Its coordinates are $w_{1}=-\varepsilon^{2}, w_{2}=0, \ldots$, $w_{n}=0$. Let

$$
\begin{aligned}
\Delta_{j}(\varepsilon)= & (-1)^{n-1} C_{n} \int_{\Omega} \frac{1}{|z-w|^{2 n}} \frac{\partial f}{\partial \bar{z}_{j}}\left(\bar{z}_{j}-\bar{w}_{j}\right) d z_{2} \cdots d z_{n} d \bar{z}_{1} \cdots d \bar{z}_{n} \\
& -(-1)^{n-1} C_{n} \int_{\Omega \backslash B(0, \varepsilon)} \frac{1}{|z|^{2 n}} \frac{\partial f}{\partial \bar{z}_{j}} \bar{z}_{j} d z_{2} \cdots d z_{n} d \bar{z}_{1} \cdots d \bar{z}_{n} \\
= & (2 i)^{n-1} C_{n} \int_{\Omega} \frac{1}{|z-w|^{2 n}}\left[\frac{\partial f}{\partial \bar{z}_{j}}(z)\left(\frac{\partial h}{\partial u_{n+1}}(z)-i\right)\right. \\
& \left.\quad-\frac{\partial f}{\partial \bar{z}_{j}}(0)\left(\frac{\partial h}{\partial u_{n+1}}(0)-i\right)\right] \\
& -(2 i)^{n-1} C_{n} \int_{\Omega \backslash B(0, \varepsilon)} \frac{1}{|z|^{2 n}}\left[\frac{\partial f}{\partial \bar{z}_{j}}(z)\left(\frac{\partial h}{\partial u_{n+1}}(z)-i\right)\right. \\
& \left.\left.\quad-\frac{\partial f}{\partial \bar{z}_{j}}(0)\left(\frac{\partial h}{\partial u_{n+1}}(0)-i\right)\right] \bar{w}_{j}\right) d u_{2} \cdots d u_{2 n} d u_{2} \cdots d u_{2 n} \\
& +(2 i)^{n-1} C_{n} \frac{\partial f}{\partial \bar{z}_{j}}(0)\left(\frac{\partial h}{\partial u_{n+1}}(0)-i\right) \int_{\Omega} \frac{\bar{z}_{j}-\bar{w}_{j}}{|z-w|^{2 n}} d u_{2} \cdots d u_{2 n} \\
& -(2 i)^{n-1} C_{n} \frac{\partial f}{\partial \bar{z}_{j}}(0)\left(\frac{\partial h}{\partial u_{n+1}}(0)-i\right) \int_{\Omega \backslash B(0, \varepsilon)} \frac{\bar{z}_{j}}{|z|^{2 n}} d u_{2} \cdots d u_{2 n} .
\end{aligned}
$$

The function $\partial h / \partial u_{n+1}-i$ is Hölder continuous. Therefore we see that when $j=2, \ldots, n$,

$$
\begin{aligned}
\left|\Delta_{j}(\varepsilon)\right| \leq & C \int_{\Omega \backslash B(0, \varepsilon)}|z|^{\alpha}\left|\frac{\bar{z}_{j}}{|z-w|^{2 n}}-\frac{\bar{z}_{j}}{|z|^{2 n}}\right| d u_{2} \cdots d u_{2 n} \\
& +C \int_{\Omega \cap B(0, \varepsilon)} \frac{|z|^{\alpha}\left|\bar{z}_{j}\right|}{|z-w|^{2 n}} d u_{2} \cdots d u_{2 n} \\
& +C \int_{\Omega \backslash B(0, \varepsilon)}\left|\frac{\bar{z}_{j}}{|z-w|^{2 n}}-\frac{\bar{z}_{j}}{|z|^{2 n}}\right| d u_{2} \cdots d u_{2 n} \\
& +C\left|\int_{\Omega \cap B(0, \varepsilon)} \frac{\bar{z}_{j}}{|z-w|^{2 n}} d u_{2} \cdots d u_{2 n}\right|
\end{aligned}
$$


By applying Lemma 3.3 we get

$$
\Delta_{j}(\varepsilon) \rightarrow 0 \text { as } \varepsilon \rightarrow 0, j=2, \ldots, n .
$$

The case $j=1$ remains to be considered:

$$
\begin{aligned}
& \left|\Delta_{1}(\varepsilon)-\frac{1}{2} A_{1} \frac{\partial f}{\partial \bar{z}_{1}}(0)\right| \leq C \int_{\Omega \backslash B(0, \varepsilon)}|z|^{\alpha}\left|\frac{\bar{z}_{1}-\bar{w}_{1}}{|z-w|^{2 n}}-\frac{\bar{z}_{1}}{|z|^{2 n}}\right| d u_{2} \cdots d u_{2 n} \\
& \quad+C \int_{\Omega \cap B(0, \varepsilon)} \frac{|z|^{\alpha}\left|\bar{z}_{1}-\bar{w}_{1}\right|}{|z-w|^{2 n}} d u_{2} \cdots d u_{2 n} \\
& +C \int_{\Omega \backslash B(0, \varepsilon)}\left|\frac{\bar{z}_{1}-\bar{w}_{1}}{|z-w|^{2 n}}-\frac{\bar{z}_{1}}{|z|^{2 n}}\right| d u_{2} \cdots d u_{2 n} \\
& +C\left|\int_{\Omega \cap B(0, \varepsilon)} \frac{\bar{z}_{1}-\bar{w}_{1}}{|z-w|^{2 n}} d u_{2} \cdots d u_{2 n}-\frac{\pi^{n}}{(n-1) !}\right| .
\end{aligned}
$$

Again by applying Lemma 3.3 we get

$$
\Delta_{1}(\varepsilon) \rightarrow \frac{1}{2} A_{1} \frac{\partial f}{\partial \bar{z}_{1}}(0) \quad \text { as } \varepsilon \rightarrow 0 .
$$

Now (3.10) follows from (3.11) and (3.12).

Now let us consider the general case. In the sequel we assume that $\Omega$ is the boundary of a domain $D$ and is of class $C^{3}$. Let $\alpha \in \Omega$ be a point. Assume that $\Omega$ is defined locally by an equation $r(z)=0$ in a neighborhood of a point $a,\left.d r\right|_{z=a} \neq 0$, so that its tangent plane at the point $a$ is

$$
\operatorname{Re} \sum_{j=1}^{n}\left(\frac{\partial r}{\partial z_{j}}\right)_{z=a}\left(z_{j}-a_{j}\right)=0 .
$$

We make a unitary transformation

$$
z-a=z^{*} U, \quad z_{k}-a_{k}=\sum_{j=1}^{n} z_{j}^{*} U_{j k},
$$

where the $U_{j k}$ satisfy

$$
\sum_{j=1}^{n} U_{k j}\left(\frac{\partial r}{\partial z_{j}}\right)_{z=a}= \begin{cases}c>0, & k=1 ; \\ 0, & k=2, \ldots, n .\end{cases}
$$

If $\Omega$ is transformed to $\Omega^{*}$, the equation for the tangent plane of $\Omega^{*}$ at $z^{*}=0$ is $c\left(z_{1}^{*}+\bar{z}_{1}^{*}\right)=0$.

Let $z_{k}^{*}=v_{k}+i v_{n+k}, k=1, \ldots, n$. Then the tangent plane of $\Omega^{*}$ at the point $z^{*}=0$ has the equation $v_{1}=0$.

Because $K_{2 n-1}(z, w)$ is invariant under the aforementioned unitary transformation, the finite part of the integral of $f$ over $\Omega$ at point $a$ can be defined as the following

$$
\begin{aligned}
& \mathrm{FP} \int_{\Omega} f(z) \frac{\bar{z}_{k}-\bar{a}_{k}}{|z-a|^{2}} K_{2 n-1}(z, a) \\
& \quad=\mathrm{FP} \int_{\Omega^{*}} \sum_{j=1}^{n} \bar{U}_{j k} f\left(z^{*} U-a\right) \frac{\bar{z}_{j}^{*}}{\left|z^{*}\right|^{2}} K_{2 n-1}\left(z^{*}, 0\right) .
\end{aligned}
$$


Now let us take

$$
U_{1 j}=\frac{\left(\partial r / \partial \bar{z}_{j}\right)_{z=a}}{\sum_{k=1}^{n}\left|\left(\partial r / \partial z_{k}\right)_{z=a}\right|^{2}} .
$$

Let $F(w)=\int_{\Omega} f(z) K(z, w)$ be the same as in Theorem 3.5. We know that

$$
\begin{aligned}
\left(\frac{\partial F}{\partial w_{k}}\right)(w) & =n \int_{\Omega} f(z) \frac{\bar{z}_{k}-\bar{w}_{k}}{|z-w|^{2}} K(z, w) \\
& =n \int_{\Omega^{*}} f\left(z^{*} U-a\right) \frac{\sum_{j=1}^{n} \bar{U}_{j k}\left(\bar{z}_{j}^{*}-\bar{w}_{j}^{*}\right)}{\left|z^{*}-w^{*}\right|^{2}} K_{2 n-1}\left(z^{*}, w^{*}\right) .
\end{aligned}
$$

By using Theorem 3.5 we see that

$$
\begin{aligned}
\left(\frac{\partial F}{\partial w_{k}}\right)_{i}(a)= & \operatorname{FP} \int_{\Omega} n f(z) \frac{\bar{z}_{k}-\bar{z}_{k}^{0}}{|z-a|^{2}} K(z, a) \\
& +\frac{1}{2} \sum_{j=1}^{n} \bar{U}_{j k} \frac{\partial f\left(z^{*} U\right)}{\partial z_{j}^{*}}(0)+\frac{1}{2} A_{1} \bar{U}_{1 k} \frac{\partial f\left(z^{*} U\right)}{\partial \bar{z}_{1}^{*}}(0) \\
= & \operatorname{FP} \int_{\Omega} n f(z) \frac{\bar{z}_{k}-\bar{z}_{k}^{0}}{|z-a|^{2}} K(z, a) \\
& +\frac{1}{2} \frac{\partial f(z)}{\partial z_{k}}(a)-\frac{1}{2} \bar{U}_{1 k} \sum_{j=1}^{n} \bar{U}_{1 j} \frac{\partial f(z)}{\partial \bar{z}_{j}}(a) .
\end{aligned}
$$

So we have proved the first one of the following formulas. The second one can be proved similarly.

$$
\begin{aligned}
\left(\frac{\partial F}{\partial w_{k}}\right)_{i}(a)= & \operatorname{FP} \int_{\Omega} n f(z) \frac{\bar{z}_{k}-\bar{a}_{k}}{|z-a|^{2}} K(z, a) \\
& +\frac{1}{2} \frac{\partial f}{\partial z_{k}}(a)-\frac{1}{2} \bar{U}_{1 k} \sum_{j=1}^{n} \bar{U}_{1 j} \frac{\partial f}{\partial \bar{z}_{j}}(a) \\
\left(\frac{\partial F}{\partial w_{k}}\right)_{e}(a)= & \operatorname{FP} \int_{\Omega} n f(z) \frac{\bar{z}_{k}-\bar{a}_{k}}{|z-a|^{2}} K(z, a) \\
& -\frac{1}{2} \frac{\partial f}{\partial z_{k}}(a)+\frac{1}{2} \bar{U}_{1 k} \sum_{j=1}^{n} \bar{U}_{1 j} \frac{\partial f}{\partial \bar{z}_{j}}(a) .
\end{aligned}
$$

Here $\left(\partial F / \partial w_{k}\right)_{i}(a)$ and $\left(\partial F / \partial w_{k}\right)_{e}(a)$ are the limits of $\left(\partial F / \partial w_{k}\right)(w), k=$ $1, \ldots, n$, as $w$ tends to $a$ along the inner normal and the exterior normal of $\Omega$ at the point $a$, respectively.

\section{REFERENCES}

C. Fox, A generalization of the Cauchy principal value, Canad. J. Math. 9 (1957), 110-117.

Sheng Gong, Singular integrals in several complex variables, Science and Technology Press, Shanghai, 1982. (Chinese)

J. Hadamard, Lectures on Cauchy's problem in linear partial differential equations, Dover, New York, 1952. 
Lars Hörmander, The analysis of linear partial differential operators. I, Springer-Verlag, 1983.

Qi-keng Lu and Tongde Zhong, A generalization of Privalov's theorem, Chinese Math. 7 (1957), 144-165. (Chinese)

Department of Mathematics, Uppsala University, P. O. Box 480, S-75106 UpPSala, SweDEN

E-mail address: Xiaoqin@math.uu.se 